\title{
Group Schemes with Additional Structures and Weyl Group Cosets
}

\author{
by Ben Moonen*
}

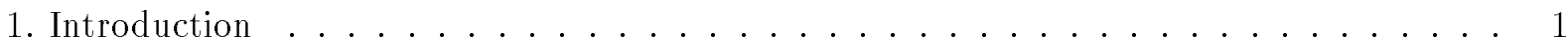

2. Review of results of Kraft .................... 3

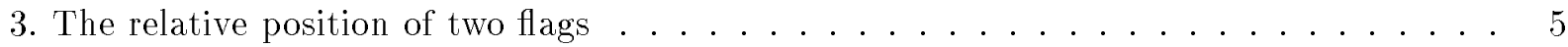

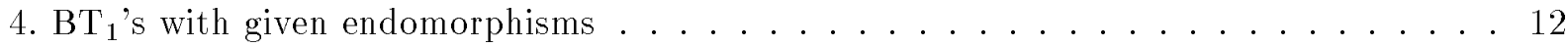

5. $\mathrm{BT}_{1}$ 's with endomorphisms and a polarization — cases $\mathrm{C}$ and $\mathrm{D} \ldots \ldots \ldots 22$

$6 . \mathrm{BT}_{1}$ 's with endomorphisms and a polarization - case A . . . . . . . . 29

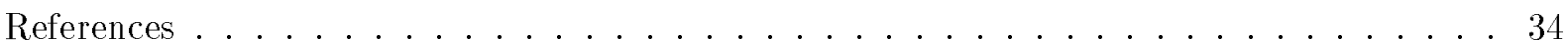

\section{Introduction}

Let $Y$ be an abelian variety of dimension $g$ over an algebraically closed field $k$ of characteristic $p>0$. To $Y$ we can associate its $p$-kernel $Y[p]$, which is a finite commutative $k$-group scheme of rank $p^{2 g}$. In the unpublished manuscript [9], Kraft showed that (fixing $g$ ) there are only finitely many such group schemes, up to isomorphism. (As we shall discuss later, Kraft also gave a very nice description of all possible types.) Some 20 years later, this result was re-obtained, independently, by Oort. Together with Ekedahl he used it to define and study a stratification of the moduli space $\mathscr{A}_{g, 1} \otimes k$ of principally polarized abelian varieties over $k$. The strata correspond to the pairs $(Y, \mu)$ such that the $p$-kernel is of a fixed isomorphism type. Their results can be found in [12] and [13]; see also related work by van der Geer in [3].

The present paper is a first step in a program to develop a similar stratification for (good reductions of) more general Shimura varieties. For Hilbert modular varieties this has been done by Goren and Oort in [7]. In general, such a Shimura variety (i.e., some $p$-adic model) should come from a $\mathbb{Z}_{(p)}$-version of a Shimura datum, involving a reductive group $G$ over $\mathbb{Z}_{(p)}$ and a homogeneous space $X$ under $G(\mathbb{R})$ such that $\left(G_{\mathbb{Q}}, X\right)$ is a Shimura datum in the usual sense. Let us suppose that there is some modular interpretation, say in terms of abelian varieties with certain prescribed Hodge/Tate classes. Now one might hope that from the data $(G, X)$ one can cook up some finite set which parametrizes the isomorphism classes of the $p$-kernels with "additional structure" that occur on the special fibre. Further one may hope that this leads to a stratification of the special fibre and that some features of the strata (say the dimensions, and which strata appear in the boundary of another one) can be described in a group theoretical way, i.e., starting from $(G, X)$.

To give meaning to such speculations, we study Shimura varieties of PEL-type. In this case at least some of the assumptions (good reduction, modular interpretation) are well understood, and we may hope to see a general pattern -if there is one-emerging from this particular case.

To explain our results, consider a PEL-type moduli problem, as stated for instance in [8], Section 5. The data involved include a simple $\mathbb{Q}$-algebra with involution $(\mathscr{D}, *)$, a $*$-stable order

\footnotetext{
* Research made possible by a fellowship of the Royal Netherlands Academy of Arts and Sciences
} 
$\mathscr{O} \subset \mathscr{D}$, a lattice $\Lambda$ equipped with an action of $\mathscr{O}$, and a symplectic form $\psi: \Lambda \times \Lambda \rightarrow \mathbb{Z}$ such that $\psi\left(d \lambda_{1}, \lambda_{2}\right)=\psi\left(\lambda_{1}, d^{*} \lambda_{2}\right)$ for $d \in \mathscr{O}$. This gives rise to a Shimura datum $\left(G_{\mathbb{Q}}, X\right)$, where $G_{\mathbb{Q}}=\operatorname{CSp}_{\mathscr{D}}\left(\Lambda_{\mathbb{Q}}, \psi\right)$. The corresponding moduli problem is, roughly, that of principally polarized abelian varieties $(Y, \mu)$ with an action of $\mathscr{O}$ such that $H_{1}(Y, \mathbb{Z}) \cong \Lambda$ as $\mathscr{O}$-modules.

Now suppose that $\mathfrak{p}$ is a prime of good reduction, of residue characteristic $p>0$. Then $D:=\mathscr{O} / p \mathscr{O}$ is a semi-simple $\mathbb{F}_{p}$-algebra and $G_{\mathbb{Q}}$ has good reduction $G$ over $\mathbb{F}_{p}$. The $p$-kernel group schemes that occur on the special fibre of the moduli space are truncated Barsotti-Tate groups $\mathscr{G}$, equipped with an action $\iota: D \rightarrow \operatorname{End}(\mathscr{G})$ and a polarization form $\varphi$ (on the Dieudonné module) such that $\varphi\left(d m_{1}, m_{2}\right)=\varphi\left(m_{1}, d^{*} m_{2}\right)$. Further, certain discrete invariants (such as the rank of $\mathscr{G}$ ) are fixed.

We show that such triples $(\mathscr{G}, \varphi, \iota)$ admit the following classification. Here we work over an algebraically closed field $k$ and we assume $p>2$. Let $W$ be the Weyl group of $G_{k}$. The space $X$ naturally gives rise to a subgroup $W_{X}$ of $W$. To a given triple $(\mathscr{G}, \varphi, \iota)$ we associate an element of $W_{X} \backslash W$, and we prove that this gives a bijection

$$
\left\{\begin{array}{c}
\text { isomorphism classes of } \\
\text { triples }(\mathscr{G}, \varphi, \iota)
\end{array}\right\} \stackrel{\sim}{\longrightarrow} W_{X} \backslash W
$$

We refer to section 4-6 for more precise statements. Note that $W_{X} \backslash W$ is a finite set which in all cases considered can be made fully explicit.

The present paper only deals with finite group schemes with additional structure. Results about the associated stratifications on PEL-type moduli spaces will appear in [10]. After that it should be investigated whether a similar group-theoretic description also applies to more general Shimura varieties. We hope to return to such questions in the near future.

Acknowledgements. I have had many pleasant conversations about the subject with Frans Oort; he explained to me the basic ideas underlying the Ekedahl-Oort stratification. That a good "encoding" could be done using the Weyl group was suggested by Gerard van der Geer (cf. his paper [3]). On several questions about algebraic groups I received useful suggestions from Wilberd van der Kallen. I thank them all cordially.

Notations and conventions. If $S$ is a set we write $\mathfrak{S}(S)$ for its group of permutations. In case $S=$ $\{1,2, \ldots, q\}$ we simply write $\mathfrak{S}_{q}$. We shall write $\mathbb{H}_{q} \subset \mathfrak{S}_{2 q}$ for the subgroup given by

$$
\mathbb{H}_{q}:=\left\{\rho \in \mathfrak{S}_{2 q} \mid \rho(j)+\rho(2 q+1-j)=2 q+1 \text { for all } j\right\}
$$

This group, isomorphic to a semi-direct product $\{ \pm 1\}^{q} \rtimes \mathfrak{S}_{q}$, is known under several names in the literature (e.g., the hyperoctahedral group, the wreath product of $\mathfrak{S}_{q}$ with $(\mathbb{Z} / 2 \mathbb{Z}$ ), ...). In this paper it will occur as (a realization of) the Weyl group of type $\mathrm{C}_{q}$.

By a symplectic form on a module $N$ we mean a perfect alternating bilinear pairing. At various points in the paper we shall work with spaces equipped with a symmetric or skew-symmetric pairing. We shall often use $\varepsilon \in\{ \pm 1\}$ for the sign of the pairing. Given a symplectic or orthogonal space $(N, \psi)$ of even dimension $2 q$ we say that an ordered $k$-basis $\beta=e_{1}, e_{2}, \ldots, e_{2 q}$ is a symplectic, resp. orthogonal basis, if

$$
\psi\left(e_{i}, e_{j}\right)= \begin{cases}0 & \text { if } i+j \neq 2 q+1 \\ 1 & \text { if } i+j=2 q+1 \text { and } i<j \\ \varepsilon & \text { if } i+j=2 q+1 \text { and } i>j\end{cases}
$$

If $\mathscr{G}$ is a finite commutative group scheme we write $\mathscr{G}^{D}$ for its Cartier dual. If $M$ is the Dieudonné module of such a group scheme, we write $M^{D}$ for the dual Dieudonné module, which is the Dieudonné module of $\mathscr{G} D$. We write $\mathscr{G}[p]$ for the kernel of multiplication by $p$. Likewise, $\mathscr{G}[F]$ and $\mathscr{G}[V]$ denote the kernels of Frobenius and Verschiebung. Similar notations $M[F]$ and $M[V]$ are used for the Dieudonné module.

If $R$ is a ring of characteristic $p>0$ then we write Frob $R: x \mapsto x^{p}$ for its Frobenius endomorphism. In the particular case that $R=k$ is a perfect field we shall occasionally write $\sigma$ for Frob $k$. 


\section{Review of results of Kraft}

2.1. Let $k$ be a perfect field of characteristic $p>0$. Write $C(1)_{k}$ for the category of finite commutative $k$-group schemes which are killed by $p$. Dieudonné theory tells us that $C(1)_{k}$ is equivalent to the category of triples $(M, F, V)$, where

- $M$ is a finite dimensional $k$-vector space,

- $F: M \rightarrow M$ is a Frob $_{k}$-linear map,

- $V: M \rightarrow M$ is a Frob ${ }_{k}^{-1}$-linear map,

such that $F \circ V=0=V \circ F$. For the purpose of this paper it is not so important how this equivalence is defined. To fix ideas, let us say we will use contravariant Dieudonné theory. A reference is [6]. In the rest of this paper, whenever we discuss Dieudonné modules we always mean modules of the kind just described, i.e., corresponding to a group scheme in the category $C(1)_{k}$.

A necessary and sufficient condition for $\mathscr{G} \in C(1)_{k}$ to be the $p$-kernel of a Barsotti-Tate group is that the sequence

$$
\mathscr{G} \stackrel{F_{\mathscr{G}}}{\longrightarrow} \mathscr{G}(p) \stackrel{V_{\mathscr{G}}}{\longrightarrow} \mathscr{G}
$$

is exact. On the Dieudonné module this means that

$$
\operatorname{Ker}(F)=\operatorname{Im}(V) \quad \text { and } \quad \operatorname{Im}(F)=\operatorname{Ker}(V) .
$$

If these conditions are satisfied we shall say that $\mathscr{G}$ is a $\mathrm{BT}_{1}$; short for: $\mathscr{G}$ is a truncated Barsotti-Tate group of level 1.

Let us now assume that $k$ is algebraically closed. In the unpublished manuscript [9], Kraft showed that the objects of $C(1)_{k}$ admit a normal form. In the description of this one distinguishes two types of group schemes.

(a) Linear type. Consider a linear graph $\Gamma$, with all edges labelled either by $F$ (in which case we draw it as $\stackrel{F}{\longrightarrow}$ ) or by $V($ drawn as $\stackrel{V}{\longleftarrow})$.

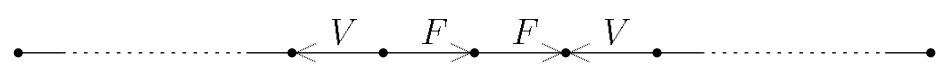

Let $\mathscr{V}=\mathscr{V}(\Gamma)$ be the set of vertices of $\Gamma$. We associate to $\Gamma$ a Dieudonné module $(M, F, V)$ in the following way. As a vector space we take $M=k^{\mathscr{Y}}$, that is, for each vertex $v$ we have a base vector $\epsilon_{v}$. Then define $F$ and $V$ according to the arrows in the graph. So, $F\left(e_{v}\right)=\epsilon_{w}$ if there is an $F$-arrow from $v$ to $w$, and $F\left(e_{v}\right)=0$ if there is not an $F$-arrow starting at $v$. Similarly, $V\left(e_{v}\right)=e_{w}$ if there is a $V$-arrow from $v$ to $w$ and $V\left(e_{v}\right)$ if $v$ is not the source of a $V$-arrow.

One readily checks that this defines a Dieudonné module, for which we write $M_{\Gamma}=$ $\left(M_{\Gamma}, F, V\right)$. Write $\mathscr{G}_{\Gamma}$ for the corresponding group scheme (well-determined up to isomorphism, or well-determined once we choose a quasi-inverse to the Dieudoné functor $\mathscr{G} \mapsto M(\mathscr{G})$ ).

(b) Circular type. Consider a circular graph

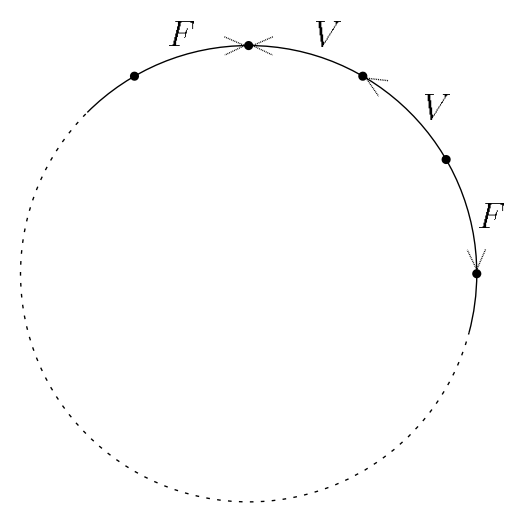


where again all edges are labelled by $F$ or $V$. We require that the $F-V$-pattern is not periodic (i.e., the diagram is not invariant under a non-trivial rotation). Two circular diagrams which differ by a rotation will be considered equivalent. By the same rules as in the linear case we obtain a Dieudonné module $M_{\Gamma}$, corresponding to a group scheme $\mathscr{G}_{\Gamma}$.

2.2. Examples. - (i) The category $C(1)_{k}$ has 3 simple objects: $(\mathbb{Z} / p \mathbb{Z}), \mu_{p}$ and $\alpha_{p}$. The corresponding diagrams are:

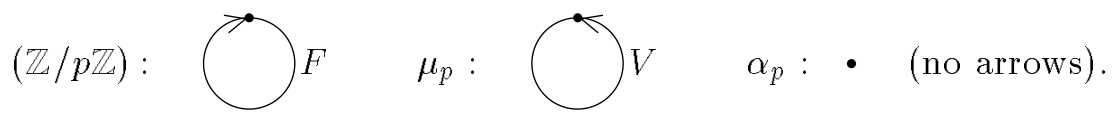

(ii) The Cartier dual of $\mathscr{G}_{\Gamma}$ is isomorphic to $\mathscr{G}_{\bar{\Gamma}}$, where $\check{\Gamma}$ is the diagram obtained from $\Gamma$ by chamging all $F$-arrows into $V$-arrows and vice versa.

(iii) The group scheme $\mathscr{G}_{\Gamma}$ is a $\mathrm{BT}_{1}$ if and only if $\Gamma$ is a circular diagram.

The category $C(1)_{k}$ is abelian, and all objects have finite length. It follows that every $\mathscr{G} \in C(1)_{k}$ is a direct sum of indecomposable objects. Up to isomorphism and permutation of the factors the indecomposables occurring in such a decomposition are uniquely determined. As the following theorem of Kraft [9] shows, the indecomposable objects are precisely the ones corresponding to the diagrams $\Gamma$ as above.

2.3. Theorem. - (i) If $\Gamma$ is a diagram of the type described above then $\mathscr{G}_{\Gamma}$ is indecomposable. If $\mathscr{G}_{\Gamma} \cong \mathscr{G}_{\Gamma^{\prime}}$ then $\Gamma$ and $\Gamma$ are equivalent, i.e., either $\Gamma=\Gamma^{\prime}$ of linear type or $\Gamma$ and $\Gamma^{\prime}$ are of circular type and differ by a rotation.

(ii) Every indecomposable object $\mathscr{G} \in C(1)_{k}$ is isomorphic to some $\mathscr{G}_{\Gamma}$.

2.4. Remark. - Let us relate the above to the description of group schemes given by Raynaud in [14]. We assume that $k=\bar{k}$. Let $\kappa$ be a finite field with $q=p^{r}$ elements. Write $\mathscr{I}:=\operatorname{Hom}(\kappa, k)$. We view this as a set with a cyclic ordering (see 4.1 below for a precise definition); given $i \in \mathscr{I}$ we write $i+1$ for the embedding $\operatorname{Frob}_{k} \circ i: \kappa \rightarrow k$. Consider pairs $(\mathscr{G}, \iota)$, and where $\mathscr{G} \in C(1)_{k}$, where $\iota: \kappa \rightarrow \operatorname{End}_{k}(\mathscr{G})$ gives $\mathscr{G}$ the structure of a scheme in $\kappa$-vector spaces. Raynaud studies the pairs $(\mathscr{G}, \iota)$ with the property that the augmentation ideal of $\mathscr{G}$ is free of rank 1 over the group ring $k\left[\kappa^{\times}\right]$. A (very) special case of loc. cit., Cor. 1.5.1. says that there is a bijection

$$
\left\{\begin{array}{l}
\text { sequences of pairs }\left(\gamma_{i}, \delta_{i}\right)_{i \in \mathscr{I}} \text { with } \\
\gamma_{i}, \delta_{i} \in\{0,1\} \text { and } \gamma_{i} \cdot \delta_{i}=0 \text { for all } i
\end{array}\right\} \stackrel{\sim}{\longrightarrow}\left\{\begin{array}{c}
\text { pairs }(\mathscr{G}, \iota) \text { up } \\
\text { to isomorphism }
\end{array}\right\}
$$

In the language of diagrams $\Gamma$, this bijection is obtained as follows. Suppose given a sequence of pairs $\left(\gamma_{i}, \delta_{i}\right)$. Take $\mathscr{I}$ as a vertex set, and draw

$$
\begin{aligned}
& \text { - an arrow }(i) \stackrel{V}{\longleftarrow}(i+1) \text { if }\left(\gamma_{i}, \delta_{i}\right)=(1,0) ; \\
& \text { - an arrow }(i) \stackrel{F}{\longrightarrow}(i+1) \text { if }\left(\gamma_{i}, \delta_{i}\right)=(0,1) ; \\
& \text { - no arrow between } i \text { and } i+1 \text { if }\left(\gamma_{i}, \delta_{i}\right)=(0,0) .
\end{aligned}
$$

This gives us a disjoint union of diagrams $\Gamma_{1}, \ldots, \Gamma_{n}$ of the type described above. The Dieudonné module $M=M_{\Gamma_{1}} \oplus \cdots \oplus M_{\Gamma_{n}}$ comes equipped with an action of $\kappa$ (letting $x \in \kappa$ act as multiplication by $i(x)$ on the base vector corresponding to $i)$, and this gives us the desired scheme in $\kappa$-vector spaces. We get a $\mathrm{BT}_{1}$ if and only if there are no pairs $\left(\gamma_{i}, \delta_{i}\right)=(0,0)$, in which case $n=1$ and $\Gamma_{1}$ is circular. (Cf. [14], Rem. 1.5.4.)

2.5. The canonical filtration. - An important tool in Kraft's proof of Theorem 2.3 is a natural filtration on the Dieudonné module $M$ of an object $\mathscr{G} \in C(1)_{k}$. This filtration is obtained, starting from $(0) \subset M$, by iterated application of the operators $F$ and $V^{-1}$. 
More formally, consider the monoid $\mathscr{M}$ with 1 freely generated by the two symbols $F$ and $V^{-1}$. On $\mathscr{U}$ we have a total (lexicographic) ordering such that

(i) $F Z<1<V^{-1} Z$ for all $Z \in \mathscr{M}$, and

(ii) if $Z_{1}<Z_{2}$ then $Z_{1} W<Z_{2} W$ for all $W \in \mathscr{M}$.

If $M^{\prime} \subset M$ is a submodule and $Z \in \mathscr{M}$ then $Z\left(M^{\prime}\right) \subset M$ is well-defined. Note that if $Z_{1}<Z_{2}$ then $Z_{1}\left(M^{\prime}\right) \subseteq Z_{2}\left(M^{\prime}\right)$. In particular, if $M^{\prime}$ is a Dieudonné submodule (i.e., stable under $F$ and $V$ ) then so is $Z\left(M^{\prime}\right)$. (Use that $F V=0=V F$.) Further, if $f: M_{1} \rightarrow M_{2}$ is a morphism of Dieudonné modules then it maps $Z\left(M_{1}\right)$ into $Z\left(M_{2}\right)$.

Now suppose that $\mathscr{G}$ is an object of $C(1)_{k}$ with Dieudonné module $M$. It follows from the properties just discussed that there is a unique coarsest filtration

$$
\mathscr{C}_{:}: \quad(0) \subsetneq \mathscr{C}_{1} \subsetneq \mathscr{C}_{2} \subsetneq \cdots \subsetneq \mathscr{C}_{r}=M
$$

of $M$ which is stable under all $Z \in \mathscr{M}$, in the sense that for every term $\mathscr{C}_{i}$ and every $Z \in \mathscr{M}$ there is an index $j$ such that $Z\left(\mathscr{C}_{i}\right)=\mathscr{C}_{j}$. This filtration $\mathscr{C}$. we call the canonical filtration of $M$. Every term $\mathscr{C}_{i}$ is of the form $\mathscr{C}_{i}=Z(0)$ or $\mathscr{C}_{i}=Z(M)$ for some $Z \in \mathscr{M}$.

2.6. Polarization forms. - Let $\mathscr{G}$ be a $\mathrm{BT}_{1}$ over $k$ with Dieudonné module $M$. As the Cartier dual $\mathscr{G}^{D}$ represents the functor $\mathscr{H} o m\left(G, \mathbb{G}_{m, k}\right)$, to give a homomorphism $\lambda: \mathscr{G} \rightarrow \mathscr{G}^{D}$ is equivalent to giving a bilinear pairing $\varphi: \mathscr{G} \times \mathscr{G} \rightarrow \mu_{p}$. On the other hand, $\lambda$ induces a morphism of Dieudonné modules $\mu: M \rightarrow M^{D}$, and this is equivalent to a $k$-bilinear form $\psi: M \times M \rightarrow k$ with $\psi\left(F m_{1}, m_{2}\right)=\psi\left(m_{1}, V m_{2}\right)^{p}$ for all $m_{1}, m_{2} \in M$.

We define $\lambda$ (or the corresponding $\mu$ ) to be a pq-polarization (short for: principal quasipolarization) if the form $\psi$ is a symplectic form, i.e., a perfect alternating pairing. If $\operatorname{char}(k) \neq$ 2 then this is equivalent to the requirement that $\lambda$ is an anti-selfdual isomorphism, i.e., an isomorphism with $\lambda^{D}=-\kappa \circ \lambda: \mathscr{G} D \rightarrow \mathscr{G} D D$, where $\kappa: \mathscr{G} \sim \sim_{\longrightarrow} \mathscr{G} D D$ is the canonical isomorphism. This, in turn, is equivalent to saying that the composition $\mathscr{G} \stackrel{\Delta}{\longrightarrow} \mathscr{G} \times \mathscr{G} \stackrel{\varphi}{\longrightarrow} \mu_{p}$ is trivial, in which case we call $\varphi$ alternating. Our motivation for using $\psi$ in the definition of a pq-polarization is the fact that for $\operatorname{char}(k)=2$ we have an implication " $\psi$ is alternating" $\Rightarrow$ " $\varphi$ is alternating", but as Oort pointed out to us, the converse does not hold in general. (A counterexample can be obtained with $\mathscr{G}$ the 2 -kernel of the supersingular elliptic curve.) Notice that if $\mathscr{G}=\widetilde{\mathscr{I}}[p]$, where $\widetilde{\mathscr{I}}$ is a Barsotti-Tate group and if $\lambda$ is induced from a quasi-polarization of $\widetilde{\mathscr{I}}$ then $\psi$ is necessarily alternating.

\section{The relative position of two flags}

In this section we discuss how the relative position of two flags in a vector space (possibly equipped with a bilinear form) can be measured by a coset in a Weyl group. First we give some abstract definitions; after that we do some concrete examples. The basic case is that of partial flags in a vector space, without further structure (see Example 3.5 below). Once this is understood, the other examples are easy variations.

Not all formulas given in the examples are needed in this paper. However, in our applications to Shimura varieties Weyl group cosets will correspond to strata in certain moduli spaces, and one may ask whether properties of these strata can be read from the corresponding coset. For instance, the dimension of the strata corresponds to the length of the reduced representative of the coset, for which we can give simple explicit formulas. On the other hand, it should be 
realized that the explicit description of the Weyl groups as permutation groups depends on choices, whereas the actual Weyl group and the elements $w^{\sharp}(P, Q)$ we consider are canonical.

3.1. We begin by reproducing some terminology and results from Bourbaki [2], Chap. 4, $\S 1$, Exercise 3 .

Let $(W, S)$ be a Coxeter system. Let $X$ and $Y$ be two subsets of $S$, and write $W_{X}$, resp. $W_{Y}$ for the subgroups of $W$ they generate. In every double class $\left(W_{X} \cdot w \cdot W_{Y}\right) \in W_{X} \backslash W / W_{Y}$ there is a unique element of minimal length, for which we write $\dot{w}$. Every other element $w^{\prime} \in W_{X} \cdot w \cdot W_{Y}$ can be written as $w^{\prime}=x \dot{w} y$ with $\ell\left(w^{\prime}\right)=\ell(x)+\ell(\dot{w})+\ell(y)$. An element $w \in W$ is said to be $(X, Y)$-reduced if it is the element of minimal length in its double class $W_{X} \cdot w \cdot W_{Y}$. The element $w$ is $(X, Y)$-reduced if and only if it is both $(X, \emptyset)$-reduced and $(\emptyset, Y)$-reduced.

3.2. Let $G$ be a connected linear algebraic group over an algebraically closed field. Fix a maximal torus and a Borel subgroup $T_{0} \subset B_{0} \subset G$. Write $W=W_{G}$ for the Weyl group, and let $S \subset W$ be the set of simple reflections corresponding to the chosen $B_{0}$. For $X \subseteq S$, write $P_{X} \subset G$ for the corresponding standard parabolic subgroup. We call a parabolic $P$ of type $X$ if $P$ is conjugate to $P_{X}$. Associating to $P$ its type gives a bijection between the set of conjugacy classes of parabolic subgroups and the powerset $\mathfrak{P}(S)$ of $S$.

The variety $\mathscr{B}$ of Borel subgroups of $G$ is homogeneous under $G$. The choice of the base point $B_{0}$ gives rise to a bijection $\varphi: W \stackrel{\sim}{\longrightarrow} G \backslash(\mathscr{B} \times \mathscr{B})$, sending $w \in W$ to the orbit of the pair $\left(B_{0},{ }^{w} B_{0}\right)$. Thus, given an ordered pair of Borel subgroups $\left(B_{1}, B_{2}\right)$ we can define $w\left(B_{1}, B_{2}\right)$ as the unique Weyl group element whose image under $\varphi$ is the orbit of $\left(B_{1}, B_{2}\right)$.

If $P$ and $Q$ are parabolic subgroups of types $X$ and $Y$, respectively, then their relative position is measured by an element

$$
w(P, Q) \in W_{X} \backslash W / W_{Y} .
$$

To define this element, choose Borel subgroups $B_{1} \subset P$ and $B_{2} \subset Q$, and let $w(P, Q)$ be the double class containing $w\left(B_{1}, B_{2}\right)$. One easily checks that this class is independent of the choice of $B_{1}$ and $B_{2}$. Alternatively, choose $g, h \in G$ such that ${ }^{g} P=P_{X}$ and ${ }^{h} Q=P_{Y}$, and let $v(P, Q) \in P_{X} \backslash G / P_{Y}$ be the class of the element $g h^{-1}$. Notice that $v(P, Q)$ is independent of the chosen elements $g$ and $h$. Then $w(P, Q)$ is the image of $v(P, Q)$ under the natural bijection $P_{X} \backslash G / P_{Y} \stackrel{\sim}{\longrightarrow} W_{X} \backslash W / W_{Y}$ obtained from Bruhat decomposition.

If we have $P$ and $Q_{1} \subset Q_{2}$ then $w\left(P, Q_{1}\right)$ maps to $w\left(P, Q_{2}\right)$ under the natural map $W_{X} \backslash W / W_{Y_{1}} \rightarrow W_{X} \backslash W / W_{Y_{2}}$. If $w$ represents $w(P, Q)$ then $w^{-1}$ represents $w(Q, P)$.

3.3. Definition. - Notations as above. We say that a parabolic subgroup $Q$ is in optimal position with respect to a parabolic subgroup $P$ if for any two Borel subgroups $B, B^{\prime} \subseteq Q$ we have $w(P, B)=w\left(P, B^{\prime}\right) \in W_{X} \backslash W$, that is, if the class $w(P, B)$ is independent of the chosen Borel subgroup $B \subseteq Q$. If $Q$ is in optimal position we write $w^{\sharp}(P, Q)$ for this class $w(P, B) \in W_{X} \backslash W$.

3.4. Remark. - The $(X, Y)$-reduced element $\dot{w}(P, Q)$ can be represented by a pair $\left(B_{1}, B_{2}\right)$ with $B_{1} \subseteq P$ and $B_{2} \subseteq Q$. Hence there exists a Borel subgroup $B \subseteq Q$ (namely $B=B_{2}$ ) such that $\dot{w}(P, B)=\dot{w}(P, Q)$. In particular, if $Q$ is in optimal position with respect to $P$ then $\dot{w}(P, B)=\dot{w}(P, Q)$ for every $B \subseteq Q$. Conversely, if this last condition holds then $Q$ is evidently in optimal position with respect to $P$.

3.5. Example $A$. - Let $N$ be a $k$-vector space of finite dimension $d$. Set $G:=\operatorname{GL}(N)$. Choose a basis $e_{1}, \ldots, e_{d}$ for $N$. Let $T_{0} \subset G$ be the diagonal torus and $B_{0} \subset G$ be the upper 
triangular Borel. We identify the Weyl group $W$ with $\mathfrak{S}_{d}$ in the usual way; the simple reflections form the set $S=\left\{s_{1}, \ldots, s_{d-1}\right\}$ with $s_{i}$ corresponding to the transposition $(i \quad i+1)$.

Let

$$
C .: \quad(0)=C_{0} \subsetneq C_{1} \subsetneq \cdots \subsetneq C_{r-1} \subsetneq C_{r}=N
$$

be a partial flag in $N$. The stabilizer of $C$. in GL(N) is a parabolic subgroup. Associating $\operatorname{Stab}\left(C_{\bullet}\right)$ to $C$. gives a bijective correspondence between partial flags in $N$ and parabolic subgroups of $G$; this allows us to apply the definitions in 3.2 and 3.3 directly to flags.

The type $Y \subseteq S$ of $C$. is given by the rule

$$
s_{i} \notin Y \quad \Longleftrightarrow \quad \exists j: \operatorname{dim}\left(C_{j}\right)=i
$$

We are interested in the relative position of a flag

$$
L: \quad(0) \subseteq L \subseteq N
$$

and a partial flag $C$. as above. If $e:=\operatorname{dim}(L)$ then $L$ has type $X=S \backslash\left\{s_{e}\right\}$ and $W_{X}$ is the subgroup $\mathfrak{S}(\{1, \ldots, e\}) \times \mathfrak{S}(\{e+1, \ldots, d\})$ of $\mathfrak{S}_{d}$. (For $e=0$ or $e=d$ this becomes $X=S$ and $W_{X}=W$.) The set $W^{\text {red }}$ of $(X, \emptyset)$-reduced elements has $\left(\begin{array}{l}d \\ e\end{array}\right)$ elements, which can be described as follows. Choose indices $1 \leq j_{1}<j_{2}<\cdots<j_{e} \leq d$, and let $1 \leq i_{1}<i_{2}<\cdots<i_{d-e} \leq d$ be the remaining ones. Given two such sequences $j$. and $i_{\bullet}$, define a permutation

$$
w=w\left(j_{\bullet}, i_{\bullet}\right) \in \mathfrak{S}_{d} \quad \text { by } \quad w\left(j_{m}\right)=m, \quad w\left(i_{m}\right)=e+m .
$$

Then $w\left(j_{\bullet}, i_{\bullet}\right)$ is an element of $W^{\text {red }}$ and every element of $W^{\text {red }}$ is of this form. The length of $w=w\left(j_{\bullet}, i_{\bullet}\right)$ is given by

$$
\ell(w)=\sum_{m=1}^{e}\left(j_{m}-m\right) .
$$

Suppose that $C_{\bullet}$ is a full flag. Set $\eta(j):=\operatorname{dim}\left(C_{j} \cap L\right)$. The sequence $\eta$ has the property that for all $j \geq 1$, either $\eta(j)=\eta(j-1)$ or $\eta(j)=\eta(j-1)+1$. Let $1 \leq j_{1}<j_{2}<\cdots<j_{e}$ be the indices with $\eta(j)=\eta(j-1)+1$, and let $1 \leq i_{1}<i_{2}<\cdots<i_{d-e}$ the indices with $\eta(i)=\eta(i-1)$. Then $\dot{w}\left(L, C_{\bullet}\right) \in \mathfrak{S}_{d}$ is the permutation $w\left(j \cdot, i_{\bullet}\right)$ as defined above.

Now let $C$. be an arbitrary partial flag. To decide whether or not $C$. is in optimal position with respect to $L$ we have to refine $C$. to a full flag $\tilde{C}$. and check whether the class $w(L, \tilde{C}$ • $)$ depends on the choice of such a refinement. By Remark 3.4 we can check this on the reduced representatives, and combined with the previous computations we find:

$$
\begin{array}{ccc}
C \text {. is in optimal position } & \Longleftrightarrow & \forall j, \text { either } C_{j} \subseteq \operatorname{Span}\left(C_{j-1}, L\right) \\
\text { with respect to } L & \text { or } C_{j-1} \cap L=C_{j} \cap L .
\end{array}
$$

3.6. Example $C$. - Let $N$ be a vector space of dimension $2 q$ over $k$, equipped with a symplectic form $\psi: N \times N \rightarrow k$. Set $G:=\operatorname{Sp}(N, \psi)$. Let $e_{1}, \ldots, e_{2 q}$ be a symplectic basis for $N$. Let $T_{0} \subset G$ be the diagonal torus and $B_{0} \subset G$ the upper triangular Borel. The Weyl group $W$ can be identified with the group

$$
\mathbb{H}_{q}:=\left\{\rho \in \mathfrak{S}_{2 q} \mid \rho(j)+\rho(2 q+1-j)=2 q+1 \text { for all } j\right\} \text {. }
$$

We have $S=\left\{s_{1}, \ldots, s_{q}\right\}$ with

$$
s_{i}=\left(\begin{array}{ll}
i & i+1
\end{array}\right) \cdot(2 q-i \quad 2 q+1-i) \quad \text { for } i \neq q, \quad \text { and } s_{q}=\left(\begin{array}{ll}
q & q+1
\end{array}\right) .
$$


A partial flag

$$
C .: \quad(0)=C_{0} \subsetneq C_{1} \subsetneq \cdots \subsetneq C_{r-1} \subsetneq C_{r}=N
$$

is called a partial symplectic flag if $\left(C_{j}\right)^{\perp}=C_{r-j}$ for all $j$. Every parabolic subgroup $Q \subset G$ is the stabilizer of a unique partial symplectic flag; as in the previous example we can therefore phrase everything directly in terms of flags. The type $Y \subseteq\left\{s_{1}, \ldots, s_{q}\right\}$ of a partial symplectic flag $C$. is given by (3.5.1).

Let $L \subset N$ be a maximal isotropic subspace. The flag $(0) \subset L \subset N$ has type $X:=S \backslash\left\{s_{q}\right\}$. We have

$$
W_{X}=\left\{\rho \in \mathbb{H}_{q} \mid \rho(\{1, \ldots, q\})=\{1, \ldots, q\}\right\} \cong \mathfrak{S}_{q} .
$$

The set $W^{\text {red }} \subset W$ of $(X, \emptyset)$-reduced elements has $2^{q}$ elements, which can be described as follows. Of each pair $\{r, 2 q+1-r\} \subset\{1,2, \ldots, 2 q\}$, choose one. Let $1 \leq j_{1}<j_{2}<\cdots<j_{q} \leq 2 q$ be the chosen elements. Let $1 \leq i_{1}<i_{2}<\cdots<i_{q} \leq 2 q$ be the elements that were not chosen, and notice that $j_{m}+i_{q+1-m}=2 q+1$ for all $m$. Define the permutation $w=w\left(i_{\bullet}, j_{\bullet}\right)$ by $w\left(j_{m}\right)=m$ and $w\left(i_{m}\right)=q+m$. (Cf. (3.5.2), where we now have $d=2 q$ and $e=q$.) Then $w$ is an element of $W^{\text {red }}$, and every element of $W^{\text {red }}$ is of this form. A reduced expression of $w$ as a word in the generators $s_{i}$ is given by

$$
w=t_{i_{l}} \cdot t_{i_{l-1}} \cdots t_{i_{1}},
$$

where $i_{l}$ is the largest among the $i_{m}$ with $i_{l} \leq q$, and where we write $t_{i}=s_{q} \cdot s_{q-1} \cdots s_{i}$. In particular, we find

$$
\ell(w)=\sum_{m=1}^{l}\left(q+1-i_{m}\right) .
$$

Consider a full symplectic flag $C_{\text {.. }}$ The reduced element $\dot{w}(L, C$. $)$ representing the class $w(L, C \cdot) \in W_{X} \backslash W$ is computed in a similar way as in 3.5. More precisely, for $0 \leq i \leq 2 q$, set $\eta(i):=\operatorname{dim}\left(C_{i} \cap L\right)$. The sequence $\eta$ has the property that for every $j \in\{1, \ldots, 2 q\}$ :

(a) either $\eta(j)=\eta(j-1)$ or $\eta(j)=\eta(j-1)+1$;

(b) $\eta(j)=\eta(j-1)$ if and only if $\eta(2 q+1-j)=\eta(2 q-j)+1$.

Let $j_{1}<j_{2}<\cdots<j_{q}$ be the indices where the sequence $\eta$ jumps and $i_{1}<i_{2}<\cdots<i_{q}$ (with $i_{m}=2 q+1-j_{q+1-m}$ ) the indices where $\eta$ does not jump. Then $\dot{w}\left(L, C_{\bullet}\right)$ is precisely the permutation $w\left(i_{\bullet}, j \bullet\right)$ defined above.

To make this concrete, take $q=7$. Let $L=\left\langle e_{1}, e_{2}, \ldots, e_{7}\right\rangle$. Let $C$. be the flag $(0) \subset$ $\left\langle e_{\pi(1)}\right\rangle \subset\left\langle e_{\pi(1)}, e_{\pi(2)}\right\rangle \subset \cdots$, where $\pi \in \mathbb{H}_{7}$. Then $\pi$ is a representative for $w(L, C \cdot)$, and the element $w$ as defined via the sequence $\eta$ is the $(X, \emptyset)$-reduced representative for the class $W_{X} \cdot \pi$. For instance, if $\pi$ is given by

$$
\pi=\left[\begin{array}{cccccccccccccc}
1 & 2 & 3 & 4 & 5 & 6 & 7 & 8 & 9 & 10 & 11 & 12 & 13 & 14 \\
2 & 11 & 12 & 7 & 9 & 5 & 1 & 14 & 10 & 6 & 8 & 3 & 4 & 13
\end{array}\right]
$$

then the sequence $\eta$ is given by

$$
\begin{array}{rccccccccccccccc}
i: & 0 & 1 & 2 & 3 & 4 & 5 & 6 & 7 & 8 & 9 & 10 & 11 & 12 & 13 & 14 \\
\eta(i): & 0 & 1 & 1 & 1 & 2 & 2 & 3 & 4 & 4 & 4 & 5 & 5 & 6 & 7 & 7
\end{array},
$$

so that $j_{1}=1, j_{2}=4, j_{3}=6, j_{4}=7, j_{5}=10, j_{6}=12, j_{7}=13 ; i_{1}=2, i_{2}=3, i_{3}=5, i_{4}=8$, $i_{5}=9, i_{6}=11, i_{7}=14$. This gives

$$
w=\dot{w}\left(L, C_{\bullet}\right)=\left[\begin{array}{cccccccccccccc}
1 & 2 & 3 & 4 & 5 & 6 & 7 & 8 & 9 & 10 & 11 & 12 & 13 & 14 \\
1 & 8 & 9 & 2 & 10 & 3 & 4 & 11 & 12 & 5 & 13 & 6 & 7 & 14
\end{array}\right],
$$


which is indeed a minimal representative (length=14) of the class $W_{X} \cdot \pi$.

Now let $C$. be a partial symplectic flag which has a maximal isotropic term (of dimension q). Combining the previous computations with Remark 3.4 we find that criterion (3.5.3) also holds in this case.

3.7. Buildings. - As preparation for the next example we need to extend the definitions in 3.2 and 3.3 to the setting of buildings. All we need from this can be found in [1] or [17]. We do not assume our buildings to be thick, that is, "building" here means "weak building" in the sense of [17].

Let $\Delta$ be a building with a strongly transitive, type-preserving action of a group $G$. Fix an apartment $\Sigma$ and a chamber $C \in \Sigma$, and let $(W, S)$ be the associated Coxeter system. (So $W$ is the group of type-preserving automorphisms of $\Sigma$.) Let $\mathfrak{p}$ and $\mathfrak{q}$ be two elements of $\Delta$, of types $X$ and $Y \subseteq S$, respectively. Let $\mathfrak{p}_{X}$ and $\mathfrak{p}_{Y}$ be the faces of $C$ of types $X$ and $Y$, and write $P_{X}:=\operatorname{Stab}_{G}\left(\mathfrak{p}_{X}\right)$ and $P_{Y}:=\operatorname{Stab}_{G}\left(\mathfrak{p}_{Y}\right)$. Bruhat decomposition gives a bijection

$$
W_{X} \backslash W / W_{Y} \stackrel{\sim}{\longrightarrow} P_{X} \backslash G / P_{Y} .
$$

Choose $g, h \in G$ with $g \cdot \mathfrak{p}=\mathfrak{p}_{X}$ and $h \cdot \mathfrak{q}=\mathfrak{p}_{Y}$. Define $w(\mathfrak{p}, \mathfrak{q}) \in W_{X} \backslash W / W_{Y}$ to be the element which, via (3.7.1), corresponds to the class of $g \cdot h^{-1}$, noting that this element does not depend on the chosen $g$ and $h$.

If $G$ is a connected reductive group and $\Delta$ is the building of (proper parabolic subgroups of) $G$ then we recover the definition given in 3.2 .

The generalization of 3.3 is immediate: we say that $\mathfrak{q}$ is in optimal position with respect to $\mathfrak{p}$ if for any two chambers $C_{1}, C_{2}$ having $\mathfrak{q}$ as a face, $w\left(\mathfrak{p}, C_{1}\right)=w\left(\mathfrak{p}, C_{2}\right)$. If this holds we obtain a well-defined element $w^{\sharp}(\mathfrak{p}, \mathfrak{q}) \in W_{X} \backslash W$.

3.8. Notation. - In the situation of 3.7 , given $X, Y \subseteq S$ we write $\mathscr{W}(\Delta ; X, Y):=$ $W_{X} \backslash W / W_{Y}$. Implicit in the notation is the choice of $C \in \Sigma \subset \Delta$, but up to a canonical isomorphism the set $\mathscr{W}(\Delta ; X, Y)$ is independent of such a choice. In the particular case that $Y=\emptyset$ we shall further abbreviate to $\mathscr{W}(\Delta ; X):=\mathscr{W}(\Delta ; X, \emptyset)=W_{X} \backslash W$. If $G$ is connected reductive and $\Delta=\Delta(G)$ then we write $\mathscr{W}(G ; X, Y)$ for $\mathscr{W}(\Delta ; X, Y)$ and $\mathscr{W}(G ; X)$ for $\mathscr{W}(\Delta ; X)$.

3.9. Example D. - Assume $\operatorname{char}(k) \neq 2$. Let $N$ be a vector space of dimension $2 q$ over $k$, equipped with a non-degenerate symmetric $k$-bilinear form $\psi: N \times N \rightarrow k$. Set $G:=\mathrm{O}(N, \psi)$.

A partial flag

$$
C .: \quad(0)=C_{0} \subsetneq C_{1} \subsetneq \cdots \subsetneq C_{r-1} \subsetneq C_{r}=N
$$

is called a partial orthogonal flag, if $\left(C_{j}\right)^{\perp}=C_{r-j}$ for all $j$. Let Flag $(N, \psi)$ be the building of all such flags, with its natural action of $G$.

Let $e_{1}, \ldots, e_{2 q}$ be an orthogonal basis for $N$. Let $\Sigma \subset \operatorname{Flag}(N, \psi)$ be the apartment of all flags $C$. with the property that each term $C_{i}$ is of the form $C_{i}=\left\langle e_{j} ; j \in J\right\rangle$ for some $J \subseteq\{1,2, \ldots, 2 q\}$. The full flag $(0) \subset\left\langle e_{1}\right\rangle \subset\left\langle e_{1}, e_{2}\right\rangle \subset \cdots \subset N$ is a chamber of $\Sigma$. The Weyl group $W$ can be identified with the group $\mathbb{H}_{q}$ as in 3.6, and the set of simple reflections is the same set $S=\left\{s_{1}, \ldots, s_{q}\right\}$ as given there. Notice that we can naturally identify $W$ with $W_{G}:=\mathscr{N}_{G}(T) / \mathscr{Z}_{G}(T)$.

Let $L \subset N$ be a maximal isotropic subspace. We are interested in the relative position of $L$ and a partial flag $C$. as above with the property that $r=2 h$ is even; in other words, the flag $C$. is assumed to have a maximal isotropic term $C_{h}$. The flag $(0) \subset L \subset N$ has type $X:=S \backslash\left\{s_{q}\right\}$. The computation of $\dot{w}(L, C \cdot)$ is now precisely the same as in 3.6 , and again we find that criterion (3.5.3) holds. 
As $G$ is non-connected, there is an alternative approach to the relative position of $L$ and $C$.. Namely, set $G^{0}:=\operatorname{SO}(N, \psi) \subset G$. Let $T_{0} \subset G^{0}$ be the diagonal maximal torus and $B_{0} \subset G^{0}$ the upper triangular Borel (with respect to the chosen orthogonal basis). Define a subgroup $\mathbb{H}_{q, \text { even }}$ and a subset $\mathbb{H}_{q, \text { odd }} \subset \mathfrak{S}_{2 q}$ by

$$
\mathbb{H}_{q, \text { even /odd }}:=\left\{\begin{array}{l|l}
\rho \in \mathfrak{S}_{2 q} & \begin{array}{l}
\rho(j)+\rho(2 q+1-j)=2 q+1 \text { for all } j, \text { and } \\
\text { the number of } i \leq q \text { with } \rho(i)>q \text { is even/odd }
\end{array}
\end{array}\right\} .
$$

The Weyl group $W^{0}$ of $G^{0}$ is $\mathbb{H}_{q, \text { even }}$. The set of simple reflections is $S^{0}=\left\{s_{1}, \ldots, s_{q-1}, \tilde{s}_{q}\right\}$, where $s_{1}, \ldots, s_{q-1}$ are as in (3.6.1) and

$$
\tilde{s}_{q}=\left(\begin{array}{ll}
q-1 & q+2
\end{array}\right) \cdot\left(\begin{array}{ll}
q & q+1
\end{array}\right) .
$$

As before, let $L \subset N$ be a totally isotropic subspace. Without loss of generality we may assume that the dimension of $\left\langle e_{1}, \ldots, e_{q}\right\rangle \cap L$ is congruent to $q$ modulo 2 , in which case the stabilizer $P \subset G^{0}$ of $L$ is a parabolic subgroup of type $X^{0}:=S \backslash\left\{\tilde{s}_{q}\right\}$. (Otherwise it is of type $S \backslash\left\{s_{q-1}\right\}$.) The set $W^{0 \text {,red }}$ of $\left(X^{0}, \emptyset\right)$-reduced elements in $W^{0}$ has $2^{q-1}$ elements, which can be described in the same spirit as in the previous examples.

Next consider a partial orthogonal flag

$$
C .: \quad(0)=C_{0} \subsetneq C_{1} \subsetneq \cdots \subsetneq C_{h} \subsetneq \cdots \subsetneq C_{2 h-1} \subsetneq C_{2 h}=N
$$

with $\operatorname{dim}\left(C_{h}\right)=q$. Let $Q \subset G^{0}$ be its stabilizer. Recall that in general an orthogonal flag is not uniquely determined by its stabilizer. (For a parabolic $Q \subset G^{0}$ there is either a unique partial orthogonal flag with stabilizer $Q$ or there are precisely 3 such flags.) A priori it is therefore not so obvious how the element $w(P, Q)$ is related to $w(L, C$.).

Now let $\delta=\delta\left(L, C_{\bullet}\right) \in(\mathbb{Z} / 2 \mathbb{Z})$ be the class of $\operatorname{dim}\left(L / L \cap C_{h}\right)$. The point we want to make is that if we know $\delta$ we can compute $w(P, Q) \in \mathscr{W}\left(G^{0} ; X^{0}\right)$ from $w(L, C \cdot) \in \mathscr{W}(F \operatorname{lag}(N, \psi) ; X)$ and vice versa. More precisely, suppose that $C$. is in optimal position with respect to $L$, working inside $\operatorname{Flag}(N, \psi)$. Notice that $\dot{w}(L, C \cdot)$ is an element of $\mathbb{H}_{q, \delta} \subset \mathbb{H}_{q}=W_{G}$. Now one can show that also $Q$ is in optimal position with respect to $P$ (in the building of $G^{0}$ ), and that

$$
w^{\sharp}(P, Q)= \begin{cases}w^{\sharp}(L, C \cdot) & \text { if } \delta \text { is even; } \\ w^{\sharp}(L, C \cdot) \cdot s_{q} & \text { if } \delta \text { is odd. }\end{cases}
$$

(Caution: the corresponding formula for reduced representatives $\dot{w}$ does not hold in general.)

In later applications to Shimura varieties the invariant $\delta$ will be fixed, and it is the element $w^{\sharp}(P, Q)$ that we have to consider. With an eye toward such applications, let us describe how the length of the reduced representative $\dot{w}(P, Q)$ can be computed, assuming that $C$. is in optimal position with respect to $L$. Namely, refine $C$. to a full orthogonal flag $\Gamma$., and set $\eta(i)=\operatorname{dim}\left(\Gamma_{i} \cap L\right)$. As usual, let the $j_{m}$ 's be the indices where the sequence $\eta$ jumps and let the $i_{m}$ 's be the remaining ones. Then

$$
\ell(\dot{w}(P, Q))=\sum_{m=1}^{l}\left(q-i_{m}\right),
$$

where $l=\operatorname{dim}\left(L / L \cap C_{h}\right)$ is the largest index such that $i_{l} \leq q$. To illustrate this, take the same data as in the example in 3.6. Then $\delta$ is odd, and

$$
\dot{w}(P, Q)=\left[\begin{array}{llllcccccccccc}
1 & 2 & 3 & 4 & 5 & 6 & 7 & 8 & 9 & 10 & 11 & 12 & 13 & 14 \\
1 & 9 & 8 & 2 & 11 & 3 & 10 & 5 & 12 & 4 & 13 & 7 & 6 & 14
\end{array}\right],
$$


which is an element of length 11 in $\mathbb{H}_{\overline{7}, \text { even }}$.

3.10. Some functoriality. - Let $G_{1} \subset G_{2}$ be an inclusion of (possibly non-connected) reductive groups. Suppose $G_{i}(i=1,2)$ acts in a strongly transitive, type-preserving way on a building $\Delta_{i}$. Further suppose given an injective map $f: \Delta_{1} \hookrightarrow \Delta_{2}$ which realizes $\Delta_{1}$ as a sub-poset of $\Delta_{2}$ and which is compatible with the actions of $G_{1}$. (The map $f$ will usually not be a morphism of complexes.) We assume that there are apartments $\Sigma_{i} \subset \Delta_{i}$ and a simplex $C$ which is a chamber of both $\Sigma_{1}$ and $\Sigma_{2}$. Write $\left(W_{i}, S_{i}\right)$ for the Coxeter system associated to $C \in \Sigma_{i} \subset \Delta_{i}$.

Let $\mathfrak{p}_{0}$ and $\mathfrak{q}_{0}$ be two faces of $C$ in $\Sigma_{1}$. Write $X_{i} \subseteq S_{i}$ for the type of $\mathfrak{p}_{0}$ and $Y_{i} \subseteq S_{i}$ for the type of $\mathfrak{q}_{0}$, working in $\Delta_{i}$. Write $P_{X_{i}}$ and $P_{Y_{i}}$ for the stabilizer of $\mathfrak{p}_{0}$ and $\mathfrak{q}_{0}$, respectively, in $G_{i}$. We have $P_{X_{1}}=G_{1} \cap P_{X_{2}}$, and $P_{Y_{1}}=G_{1} \cap P_{Y_{2}}$. This gives us a diagram

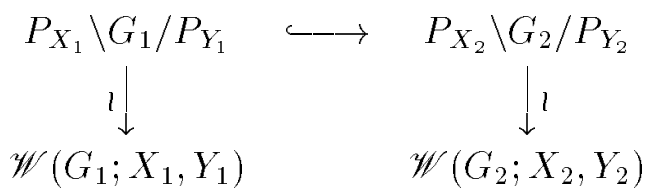

where the vertical bijections are obtained from Bruhat decomposition. Write

$$
\mathscr{W}(f): \mathscr{W}\left(G_{1} ; X_{1}, Y_{1}\right) \longleftrightarrow \mathscr{W}\left(G_{2} ; X_{2}, Y_{2}\right)
$$

for the injective map thus obtained.

Now let $\mathfrak{p}$ be an element in the $G_{1}$-orbit of $\mathfrak{p}_{0}$ and $\mathfrak{q}$ in the $G_{1}$-orbit of $\mathfrak{q}_{0}$. Viewing $\mathfrak{p}$ and $\mathfrak{q}$ as elements of $\Delta_{1}$ we obtain an element $w_{1}(\mathfrak{p}, \mathfrak{q}) \in \mathscr{W}\left(\Delta_{1} ; X_{1}, Y_{1}\right)$; viewing them as elements of $\Delta_{2}$ gives $w_{2}(\mathfrak{p}, \mathfrak{q}) \in \mathscr{W}\left(\Delta_{2} ; X_{2}, Y_{2}\right)$. Now it is immediate from the definitions that $w_{2}(\mathfrak{p}, \mathfrak{q})$ is the image of $w_{1}(\mathfrak{p}, \mathfrak{q})$ under the map $\mathscr{W}(f)$.

As an example of this, let $G_{1}=\operatorname{Sp}(N, \psi)$ as in 3.6 or $G_{1}=\mathrm{O}(N, \psi)$ as in 3.9, and consider the natural inclusion map $G_{1} \subset G_{2}:=\operatorname{GL}(N)$. There is a natural forgetful map $f: F \operatorname{lag}(N, \psi) \longleftrightarrow F \operatorname{lag}(N)$. Let $L$ and $C$. as in 3.6 or 3.9. Write $X^{\prime}$ for the type of $L$ in Flag $(N)$ (=the building of GL( $N)$ ). Then our computations show that, indeed, the answer for $w\left(L, C_{\bullet}\right)$ is the same whether we compute it in $\mathscr{W}(\operatorname{Flag}(N, \psi) ; X)$ or in $\mathscr{W}\left(F \operatorname{lag}(N) ; X^{\prime}\right)$. Even better, in both examples we have a commutative diagram

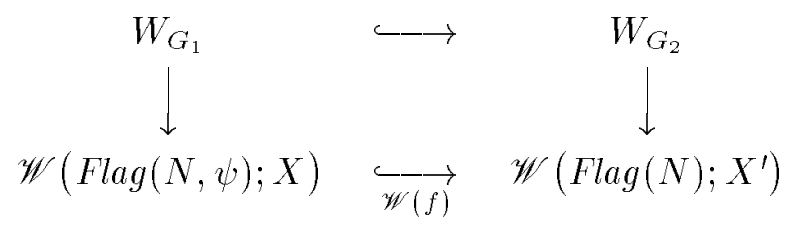

and the reduced representative $\dot{w}(L, C \cdot)$ is the same whether we compute it in $W_{G_{1}}$ or in $W_{G_{2}}$. (Of course, the length of this element in general does depend on the group in which we work.)

3.11. Example A (continued). - Since Type A will occur as a unitary group, let us discuss what happens under duality. Let $\tilde{k}:=k \times k$, with involution given by $\left(x_{1}, x_{2}\right) \mapsto\left(x_{2}, x_{1}\right)$. Let $N$ be a free $\tilde{k}$-module of rank $q$, equipped with a non-degenerate alternating hermitian form $\psi: N \times N \rightarrow \tilde{k}$. We have a natural decomposition $N=N_{1} \oplus N_{2}$, where $\left(x_{1}, x_{2}\right) \in \tilde{k}$ acts on $N_{i}$ as multiplication by $x_{i}$. The form $\psi$ gives an identification of $N_{2}$ with the $k$-linear dual of $N_{1}$. Given a subspace $L_{1} \subset N_{1}$, we write

$$
L_{1}^{\perp}:=\left\{n_{2} \in N_{2} \mid \psi\left(n_{1}, n_{2}\right)=0 \text { for all } n_{1} \in L\right\} \subset N_{2} .
$$


More generally, if $C_{1}, \bullet$ is a partial flag in $N_{1}$, we write $C_{1,}^{\perp}$ for the partial flag in $N_{2}$ whose terms are the spaces $C_{1, i}^{\perp}$ (with reversed numbering).

Let $G:=\mathrm{U}_{\tilde{k}}(N, \psi)$. We have isomorphisms

$$
\mathrm{GL}_{k}\left(N_{1}\right) \stackrel{\sim}{\longrightarrow} G \stackrel{\sim}{\longrightarrow} \mathrm{GL}_{k}\left(N_{2}\right)
$$

Under the identification $N_{2}=N_{1}^{\vee}$ the composition of the two maps is given by $g \mapsto\left(g^{\vee}\right)^{-1}$.

Choose $k$-bases $e_{i, 1}, \ldots, e_{i, q}$ for $N_{i}(i=1,2)$ such that $\psi\left(e_{1, \mu}, e_{2, \nu}\right)=0$ whenever $\mu+\nu \neq$ $q+1$. Write $T_{i} \subset B_{i} \subset \mathrm{GL}\left(N_{i}\right)$ for the diagonal torus and the upper triangular Borel. As in Example 3.5 we identify the Weyl group $W_{i}$ of GL( $\left(N_{i}\right)$ with $\mathfrak{S}_{q}$, and number the set $S_{i} \subset W_{i}$ of simple reflections such that (3.5.1) holds.

Let $w_{0} \in \mathfrak{S}_{q}$ be the permutation of order 2 given by $w_{0}(\mu)=q+1-\mu$. For $\pi \in \mathfrak{S}_{q}$, define $\check{\pi}:=w_{0} \circ \pi \circ w_{0}$. We can describe the Weyl group of $G$ as

$$
W=\left\{\left(\pi_{1}, \pi_{2}\right) \in W_{1} \times W_{2} \mid \pi_{2}=\check{\pi}_{1}\right\}
$$

where the simple reflections are the elements $\left(s_{\mu}, s_{\nu}\right) \in S_{1} \times S_{2}$ with $\mu+\nu=q+1$. For all computational purposes we can reduce to the case $\mathrm{GL}_{q}$ and proceed as in 3.5 , but up to a duality $\pi \mapsto \tilde{\pi}$ the answers obtained depend on which of the two projections $\operatorname{pr}_{i}: G \stackrel{\sim}{\longrightarrow} \operatorname{GL}\left(N_{i}\right)$ we use.

More specifically, suppose $L \subset N$ is a maximal isotropic subspace, i.e., $L=L_{1} \oplus L_{2}$ with $L_{2}=L_{1}^{\perp}$. Let $C_{1}$, be a partial flag in $N_{1}$ and $C_{2}, \bullet:=C_{1, \bullet}^{\perp}$ the "dual" partial flag in $N_{2}$. Let $\operatorname{dim}\left(L_{1}\right)=e$, so that the flag $(0) \subset L_{1} \subset N_{1}$ has type $X_{1}:=S_{1} \backslash\left\{s_{e}\right\}$ and the flag $(0) \subset L_{2} \subset N_{2}$ has type $X_{2}:=S_{2} \backslash\left\{s_{q-e}\right\}$. We have a well-defined bijection $\beta: W_{X_{1}} \backslash W_{1} \stackrel{\sim}{\longrightarrow} W_{X_{2}} \backslash W_{2}$ by $\pi \mapsto \tilde{\pi}$. Assume that $C_{i}$, is in optimal position with respect to $L_{i}$; if this holds for one $i$ then also for the other. Then $\dot{w}\left(L_{2}, C_{2}, \bullet\right)=\dot{w}\left(L_{1}, C_{1}, \bullet\right)^{\vee}$ and $\beta$ sends the class $w\left(L_{1}, C_{1}, \bullet\right)$ to the class $w\left(L_{2}, C_{2}, \bullet\right)$.

\section{4. $\mathrm{BT}_{1}$ 's with given endomorphisms}

We now start working on the subject proper of this paper: classification of finite group schemes with additional structure. In this section we deal with $\mathrm{BT}_{1}$ 's equipped with an action of a finite dimensional semi-simple $\mathbb{F}_{p}$-algebra-see problem (GE) in 4.2 for a precise statement. Although it does not "correspond" to a Shimura variety (as we do not consider polarization forms), we may view (GE) as the "basic" problem. Understanding this case will make the polarized case (GPE) considered in the next section much more transparent.

After some reduction steps, a solution of problem (GE) is given in Theorem 4.7. The proof will occupy most of the rest of the section.

4.1. Definition. - Let $\mathscr{I}$ be a finite set, say of cardinality $m$. By a cyclic ordering of $\mathscr{I}$ we mean an equivalence class of bijections $(\mathbb{Z} / m \mathbb{Z}) \rightarrow \mathscr{I}$, two such bijections being equivalent if they differ by a translation in $(\mathbb{Z} / m \mathbb{Z})$. If $m$ is even, say $m=2 n$ then by a bi-cyclic ordering of $\mathscr{I}$ we mean an equivalence class of bijections $(\mathbb{Z} / n \mathbb{Z}) \times(\mathbb{Z} / n \mathbb{Z}) \rightarrow \mathscr{I}$, where two such bijections are equivalent if they differ by a translation over an element $(a, a)$ in $(\mathbb{Z} / n \mathbb{Z}) \times(\mathbb{Z} / n \mathbb{Z})$.

If $m=2 n$ is even then a cyclic or bi-cyclic ordering of $\mathscr{I}$ gives rise to an involution of $\mathscr{I}$, denoted $\tau \mapsto \tilde{\tau}$. Namely, if $\gamma:(\mathbb{Z} / 2 n \mathbb{Z}) \rightarrow \mathscr{I}$ defines the cyclic ordering, resp. if $\gamma:(\mathbb{Z} / n \mathbb{Z}) \times$ $(\mathbb{Z} / n \mathbb{Z}) \rightarrow \mathscr{I}$ defines the bi-cyclic ordering, set

$$
\widetilde{\gamma(a)}=\gamma(a+n) \quad \text { (cyclic case), } \quad \text { resp. } \quad \gamma\left(\widetilde{a_{1}, a_{2}}\right)=\gamma\left(a_{2}, a_{1}\right) \quad \text { (bi-cyclic case) }
$$


This defines a map $\tau \mapsto \tilde{\tau}$, independent of the chosen representative $\gamma$ and compatible with the (bi-)cyclic ordering.

4.2. The setup. - Let $k$ be an algebraically closed field of characteristic $p>0$. Let $D$ be a finite dimensional semi-simple $\mathbb{F}_{p}$-algebra. The first problem that we are interested in is the following:

(GE) Classification of pairs $(\mathscr{G}, \iota)$, where $\mathscr{G}$ is a $\mathrm{BT}_{1}$ over $k$ and $\iota: D \rightarrow$ End $_{k}(\mathscr{G})$ defines an action of $D$ on $\mathscr{G}$ (with $\iota(1)=\mathrm{id} \mathscr{G})$.

As the Brauer group of a finite field is trivial we have $D \cong M_{r_{1}}\left(\kappa_{1}\right) \times \cdots \times M_{r_{n}}\left(\kappa_{n}\right)$, where the $\kappa_{i}$ are finite fields and $r_{1}, \ldots, r_{n} \in \mathbb{Z}_{\geq 1}$. Fixing such an isomorphism, every pair $(\mathscr{G}, \iota)$ as in $(\mathrm{GE})$ is of the form

$$
(\mathscr{G}, \iota)=\left(\mathscr{H}_{1}^{r_{1}}, \theta_{1}^{r_{1}}\right) \times \cdots \times\left(\mathscr{H}_{n}^{r_{n}}, \theta_{n}^{r_{n}}\right)
$$

where $\left(\mathscr{H}_{i}, \theta_{i}\right)$ is a $\mathrm{BT}_{1}$ with an action of $\kappa_{i}$, and where we write $\theta^{r_{i}}$ for the induced action of $M_{r_{i}}\left(\kappa_{i}\right)$ on $\mathscr{H}_{i}^{r_{i}}$. Thus, in dealing with problem (GE) we may from now on assume that $D=\kappa$ is a finite field.

Set $\mathscr{I}:=\operatorname{Hom}(k, k)$. This set comes naturally equipped with a cyclic ordering, where we take the successor of an embedding $i: k \rightarrow k$ to be Frob $_{k} \circ i$. If there is no risk of confusion we simply write $i+1$ for the successor of the element $i \in \mathscr{I}$.

Write

$$
k_{i}:=\kappa \otimes k \quad \text { and } \quad K:=\kappa \otimes \mathbb{F}_{p} k=\prod_{i \in \mathscr{I}} k_{i} .
$$

Of course, $k_{i}$ is canonically isomorphic to $k$; the index $i$ just reminds us of how $k$ acts. We have a Frob $k_{k}$-linear isomorphism

$$
F_{k_{i}}:=\mathrm{id} \otimes \operatorname{Frob}_{k}: k_{i} \rightarrow k_{i+1} \text {. }
$$

4.3. Decomposition of the Dieudonné module. - Let $(\mathscr{H}, \theta)$ be a $\mathrm{BT}_{1}$ with $\kappa$-action. Write $N$ for the (covariant) Dieudonné module of $\mathscr{H}$. We can view $N$ as a module over $K$. There is a natural decomposition

$$
N=\underset{i \in \mathscr{I}}{\oplus} N_{i}, \quad \text { with } N_{i}:=\{n \in N \mid a(n)=i(a) \cdot n \text { for all } a \in \kappa\} \text {. }
$$

The Frobenius and Verschiebung $F_{N}$ and $V_{N}$ restrict to homomorphisms

$$
F_{N_{i}}: N_{i} \rightarrow N_{i+1} \quad \text { and } \quad V_{N_{i}}: N_{i} \leftarrow N_{i+1} \text {, }
$$

equivariant with respect to $F_{k_{i}}: k_{i} \rightarrow k_{i+1}$, respectively its inverse. The maps $F_{N_{i}}$ and $V_{N_{i}}$ satisfy

$$
\operatorname{Ker}\left(F_{N_{i}}\right)=\operatorname{Im}\left(V_{N_{i}}\right) \quad \text { and } \quad \operatorname{Ker}\left(V_{N_{i}}\right)=\operatorname{Im}\left(F_{N_{i}}\right)
$$

This last relation gives (simply writing $F$ for $F_{N}$ and $V$ for $V_{N}$ )

$$
\begin{aligned}
\operatorname{dim}_{k}\left(N_{i}\right) & =\operatorname{dim}_{k}\left(\operatorname{Ker}\left(F_{\mid N_{i}}\right)\right)+\operatorname{dim}_{k}\left(F N_{i}\right) \\
& =\operatorname{dim}_{k}\left(V N_{i+1}\right)+\operatorname{dim}_{k}\left(\operatorname{Ker}\left(V_{\mid N_{i+1}}\right)\right)=\operatorname{dim}_{k}\left(N_{i+1}\right) .
\end{aligned}
$$

As the index set $\mathscr{I}$ has a cyclic ordering we conclude that $d:=\operatorname{dim}\left(N_{i}\right)$ is independent of $i \in \mathscr{I}$, so that $N$ is free of rank $d$ as a $K$-module. 
4.4. Canonical filtrations. - Let

$$
\mathscr{C}_{:}: \quad(0)=\mathscr{C}_{0} \subsetneq \mathscr{C}_{1} \subsetneq \cdots \subsetneq \mathscr{C}_{l}=N
$$

be the canonical filtration of $N$, introduced in 2.5. As each $\mathscr{C}_{\nu} \subset N$ is a $K$-submodule, it is of the form $\mathscr{C}_{\nu}=\oplus \Gamma_{i}$, where $\Gamma_{i}$ is a $k_{i}$-submodule of $N_{i}$. Let

$$
C_{i,:} \quad(0)=C_{i, 0} \subsetneq C_{i, 1} \subsetneq \cdots \subsetneq C_{i, \ell_{i}}=N_{i}
$$

be the filtration by $k_{i}$-submodules of $N_{i}$ thus obtained. Note that we take the inclusions in this filtration to be strict; hence the projection of $\mathscr{C}_{\nu}$ to $N_{i}$ is of the form $C_{i, j}$ with in general $j \neq \nu$.

By construction, for each $i \in \mathscr{I}$ there is an index $h_{i} \in\left\{0,1, \ldots, \ell_{i}\right\}$ such that $C_{i, h_{i}}=$ $\operatorname{Im}\left(F_{N_{i-1}}\right)$.

4.5. Lemma. - (i) For all $i \in \mathscr{I}$ and $j \in\left\{0,1, \ldots, \ell_{i}\right\}$ there exist indices $r(j)=r_{i}(j) \in$ $\left\{0,1, \ldots, h_{i+1}\right\}$ and $s(j)=s_{i}(j) \in\left\{h_{i+1}, \ldots, \ell_{i+1}\right\}$ such that

$$
F_{N_{i}}\left(C_{i, j}\right)=C_{i+1, r(j)} \quad \text { and } \quad V_{N_{i}}^{-1}\left(C_{i, j}\right)=C_{i+1, s(j)} .
$$

(ii) If $j \geq 1$ then either

$$
C_{i, j} \cap \operatorname{Ker}\left(F_{N_{i}}\right)=C_{i, j-1} \cap \operatorname{Ker}\left(F_{N_{i}}\right),
$$

or

$$
C_{i, j} \subseteq \operatorname{Span}\left(C_{i, j-1}, \operatorname{Ker}\left(F_{N_{i}}\right)\right) .
$$

In the first case we have $r_{i}(j)=r_{i}(j-1)+1$ and $s_{i}(j)=s_{i}(j-1)$; in the second case $r_{i}(j)=$ $r_{i}(j-1)$ and $s_{i}(j)=s_{i}(j-1)+1$.

(iii) The integer $\ell:=\ell_{i}$ does not depend on $i \in \mathscr{I}$. For all $i$ and $j$ we have $r_{i}(j)+s_{i}(j)=$ $h_{i+1}+j$.

Proof. Statement (i) follows from the fact that the canonical filtration $\mathscr{C}$. is (by construction) stable under $F_{N}$ and $V_{N}^{-1}$, using that $F_{N}$ and $V_{N}$ commute with the action of $\kappa$. Assertion (ii) follows from the definition of the canonical filtration together with (4.3.1). It follows from (ii) that $r_{i}(j)+s_{i}(j)=r_{i}(j-1)+s_{i}(j-1)+1$ for all $j \geq 1$. Noting that $r_{i}(0)=0$ and $s_{i}(0)=h_{i+1}$ we find that $r_{i}(j)+s_{i}(j)=h_{i+1}+j$. On the other hand, $r_{i}\left(\ell_{i}\right)=h_{i+1}$ and $s_{i}\left(\ell_{i}\right)=\ell_{i+1}$. This gives that $\ell_{i}=\ell_{i+1}$.

4.6. Definition of the element $\underline{w}(\mathscr{H}, \theta)$. - For $i \in \mathscr{I}$, let $\mathfrak{f}(i)$ be the $k$-dimension of $N[F]_{i}:=\operatorname{Ker}\left(F_{N_{i}}\right)$. This defines a function

$$
\mathfrak{f}: \mathscr{I} \rightarrow\{0,1, \ldots, d\}
$$

which (by analogy to the classical notion of a CM-type) we refer to as the multiplication type of the pair $(\mathscr{H}, \theta)$.

The integer $d:=\operatorname{rk}_{K}(N)$ and the function $\mathfrak{f}$ should be thought of as "discrete invariants" of the pair $(\mathscr{H}, \theta)$, and for problem (GE) we may assume them to be fixed. To state the theorem below we want to rigidify $N$, i.e., choose an isomorphism $\xi: N \stackrel{\sim}{\longrightarrow} K^{d}$. Recall that $K=\prod_{i \in \mathscr{I}} k_{i}$.

Let $G:=\mathrm{GL}_{d, K}=\prod_{i \in \mathscr{I}} G_{i}$, with $G_{i}=\mathrm{GL}_{d, k_{i}}$. The function $\mathfrak{f}$ determines a conjugacy class $X=X_{f}$ of parabolic subgroups of $G$, viz., the conjugacy class containing the stabilizer 
of $N[F] \subset N \stackrel{\sim}{\longrightarrow} K^{d}$. The type $X$ decomposes as a "product" of types $X_{i}$ in the factors $G_{i}$; identifying the Weyl group of $G_{i}$ with $\mathfrak{S}_{d}$ as in 3.5 we have $X_{i}=S \backslash\left\{s_{f(i)}\right\}$.

Via the chosen isomorphism $\xi$ we can view $N[F]_{i}$ and $C_{i}$, as flags in $k_{i}^{d}$. Combining (ii) of the lemma with (3.5.3), we find that each $C_{i}$, is in optimal position with respect to $N[F]_{i}$. This gives us a well-defined element $w^{\sharp}\left(N[F]_{i}, C_{i}, \bullet\right) \in \mathscr{W}\left(G_{i} ; X_{i}\right)$. Now define

$$
\underline{w}(\mathscr{H}, \theta) \in \mathscr{W}(G ; X)=\prod_{i \in \mathscr{I}} \mathscr{W}\left(G_{i} ; X_{i}\right)
$$

to be the element with $w_{i}(\mathscr{H}, \theta)=w^{\sharp}\left(N[F]_{i}, C_{i}, \bullet\right)$. Alternatively, since each $C_{i}, \bullet$ is in optimal position, it follows that also $Q:=\operatorname{Stab}_{G}\left(\mathscr{C}_{\bullet}\right)$ is in optimal position with respect to $P:=$ $\operatorname{Stab}_{G}(N[F])$, and $\underline{w}(\mathscr{H}, \theta)$ is just the element $w^{\sharp}(P, Q)$. One easily checks that this element does not depend on the choice of $\xi$. Further it is clear that isomorphic pairs $(\mathscr{H}, \theta)$ give the same element $\underline{w}(\mathscr{H}, \theta)$.

4.7. Theorem. - Notations and assumptions as in 4.2 and 4.6. Sending $(\mathscr{H}, \theta)$ to the element $\underline{w}(\mathscr{H}, \theta)$ gives a bijection

$$
\left\{\begin{array}{c}
\text { isomorphism classes of pairs } \\
(\mathscr{H}, \theta) \text { of type }(d, \mathfrak{f})
\end{array}\right\} \stackrel{\sim}{\longrightarrow} \mathscr{H}(G ; X) \cong \prod_{i \in \mathscr{I}}\left[\mathfrak{S}_{\mathfrak{f}(i)} \times \mathfrak{S}_{d-\mathfrak{f}(i)} \backslash \mathfrak{S}_{d}\right] .
$$

(In the last formula, we have written $\mathfrak{S}_{\mathfrak{f}(i)} \times \mathfrak{S}_{d-\mathfrak{f}(i)}$ as an abbreviation for $\mathfrak{S}(\{1, \ldots, \mathfrak{f}(i)\}) \times$ $\mathfrak{S}(\{\mathfrak{f}(i)+1, \ldots, d\})$.)

The proof of the theorem will occupy the rest of this section.

4.8. Standard objects. - We use the notations introduced in 4.6. Let $\underline{w}=\left\{w_{i}\right\}_{i \in \mathscr{I}} \in$ $\mathscr{W}(G ; X)=\prod_{i \in \mathscr{I}} \mathscr{W}\left(G_{i} ; X_{i}\right)$. We shall define a pair $(\mathscr{H}, \theta)=\left(\mathscr{H}_{\underline{w}}, \theta_{\underline{w}}\right)$ of type $(d, \mathfrak{f})$ such that $\underline{w}(\mathscr{H}, \theta)=\underline{w}$. We shall refer to this pair as the standard object corresponding to $\underline{w}$.

For $i \in \mathscr{I}$, let $e_{i, 1}, \ldots, e_{i, d}$ be the standard basis of $k_{i}^{d}$. We use the explicit description of $\mathscr{W}\left(G_{i} ; X_{i}\right)$ given in 3.5 ; in particular we identify the Weyl group of $G_{i}$ with $\mathfrak{S}_{d}$. Consider the $\left(X_{i}, \emptyset\right)$-reduced representatives $\dot{w}_{i} \in \mathfrak{S}_{d}$. Now define $F_{i}: k_{i}^{d} \rightarrow k_{i+1}^{d}$ and $V_{i}: k_{i}^{d} \leftarrow k_{i+1}^{d}$ to be the Frob $_{k}$-linear, resp. Frob ${ }_{k}^{-1}$-linear maps given on the base vectors $e_{i, j}$ by

$$
F_{i}\left(e_{i, j}\right)= \begin{cases}0 & \text { if } \dot{w}_{i}(j) \leq \mathfrak{f}(i) \\ e_{i+1, m} & \text { if } \dot{w}_{i}(j)=\mathfrak{f}(i)+m\end{cases}
$$

and

$$
V_{i}\left(e_{i+1, j}\right)= \begin{cases}0 & \text { if } j \leq d-\mathfrak{f}(i) \\ e_{i, n} & \text { if } j=d-\mathfrak{f}(i)+\dot{w}_{i}(n) .\end{cases}
$$

One readily checks that this gives $K^{d}$ the structure of a Dieudonné module corresponding to a pair $\left(\mathscr{H}_{\underline{w}}, \theta_{\underline{w}}\right)$ of type $(d, \mathfrak{f})$. We shall write $N_{\underline{w}}$ for the Dieudonné module thus constructed. For each $i$ the full flag

$$
E_{i,:} \quad(0) \subset\left\langle e_{i, 1}\right\rangle \subset\left\langle e_{i, 1}, e_{i, 2}\right\rangle \subset \cdots \subset k_{i}^{d}
$$

is a refinement of the canonical filtration $C_{i}, \bullet$ Hence $w^{\sharp}\left(\operatorname{Ker}\left(F_{i}\right), C_{i}, \bullet\right)=w\left(\operatorname{Ker}\left(F_{i}\right), E_{i}, \bullet\right)$. Computing $\dot{w}\left(\operatorname{Ker}\left(F_{i}\right), E_{i}, \bullet\right)$ following the recipe in 3.5 we precisely find back the element $\underline{w}$. Hence $\underline{w}\left(\mathscr{H}_{\underline{w}}, \theta_{\underline{w}}\right)=\underline{w}$, as desired. 
4.9. The canonical blocks. - We shall freely use the notations introduced in Lemma 4.5. We refer to the $k$-vector spaces

$$
B_{i, j}:=C_{i, j} / C_{i, j-1} \quad(\text { for } i \in \mathscr{I} \text { and } j \in\{1, \ldots, \ell\})
$$

as the canonical blocks. We define $\rho_{i}:\{1, \ldots, \ell\} \rightarrow\{1, \ldots, \ell\}$ by

$$
\rho_{i}(j)= \begin{cases}r_{i}(j) & \text { if } C_{i, j} \cap \operatorname{Ker}\left(F_{N_{i}}\right)=C_{i, j-1} \cap \operatorname{Ker}\left(F_{N_{i}}\right) ; \\ s_{i}(j) & \text { if } C_{i, j} \subseteq \operatorname{Span}\left(C_{i, j-1}, \operatorname{Ker}\left(F_{N_{i}}\right)\right) .\end{cases}
$$

It follows from Lemma 4.5 that $\rho_{i}$ is a permutation.

For each $i \in \mathscr{I}$ and $j \in\{1,2, \ldots, \ell\}$ we obtain a canonical Frob $_{k}$-linear isomorphism

$$
t_{i, j}: B_{i, j} \stackrel{\sim}{\longrightarrow} B_{i+1, \rho_{i}(j)},
$$

induced by the Frobenius $F_{N_{i}}: C_{i, j} \rightarrow C_{i+1, r_{i}(j)}$ if $r_{i}(j)>r_{i}(j-1)$ or (inversely) by the Verschiebung $V_{N_{i}}: C_{i, j} \leftarrow C_{i+1, s_{i}(j)}$ if $s_{i}(j)>s_{i}(j-1)$. In the first case we refer to $t_{i, j}$ as an $F$-arrow, in the second case we call it a $V$-arrow.

In what follows it will be important to follow how the blocks $B_{i, j}$, thought of as the building blocks of the modules $N_{i}$, "migrate" through the circularly ordered system of modules $N_{i}$. (For an illustration of this, see 4.11 below.) We shall use the following notations and terminology.

Write $\mathscr{A}:=\mathscr{I} \times\{1, \ldots, \ell\}$. Let $\rho$ be the permutation of $\mathscr{A}$ given by $\rho(i, j)=\left(i+1, \rho_{i}(j)\right)$. By an orbit in $\mathscr{A}$ we simply mean an orbit under the permutation $\rho$. It will often be convenient to denote an element of $\mathscr{A}$ by a single letter (usually $a$ ). Thus, if $a=(i, j) \in \mathscr{A}$, we shall write $t_{a}: B_{a} \rightarrow B_{\rho(a)}$ for the map $t_{i, j}$ above, etc.

4.10. Lemma. - For every $a \in \mathscr{A}$ we can choose an ordered basis $\beta_{a}$ for $B_{a}$ such that $\beta_{\rho(a)}$ is the image of $\beta_{a}$ under $t_{a}: B_{a} \rightarrow B_{\rho(a)}$.

Proof. Clearly it suffices to find the desired bases for $a$ running through one $\rho$-orbit $O \subset \mathscr{A}$. Notice that $O$ is a finite set with a natural cyclic ordering. Let $m:=\# O$ and choose $a(0) \in O$. Write $a(n):=\rho^{n}(a(0))$, so that $a(m)=a(0)$. The composition

$$
\mathscr{T}:\left(B_{a(0)} \stackrel{t_{a(0)}}{\longrightarrow} B_{a(1)} \stackrel{t_{a(1)}}{\longrightarrow} \cdots \stackrel{t_{a(m-1)}}{\longrightarrow} B_{a(m)}=B_{a(0)}\right)
$$

is a Frob $_{k}^{m}$-linear bijection. Then

$$
\mathscr{B}_{a(0)}:=\left\{b \in B_{a(0)} \mid \mathscr{T}(b)=b\right\}
$$

is an $\mathbb{F}_{p^{m}}$-subspace of $B_{a(0)}$ such that the natural map $\mathscr{B}_{a(0)} \otimes_{\mathbb{T}_{p} m} k \longrightarrow B_{a(0)}$ is an isomorphism. Now choose an ordered $\mathbb{F}_{p^{m}}$-basis $\beta_{a(0)}$ for $\mathscr{B}_{a(0)}$, and define $\beta_{a(n)}$ to be the $\rho^{n}$-image of $\beta_{a(0)}$.

4.11. Remark. - Consider the $K$-module $L:=\oplus_{(i, j) \in \mathscr{A}} B_{i, j}$, where $c \in \kappa$ acts on a summand $B_{i, j}$ as multiplication by $i(c)$. Let $b \in B_{i, j}$. Define

$$
F(b):= \begin{cases}t_{i, j}(b) & \text { if } r_{i}(j)>r_{i}(j-1) \\ 0 & \text { if } r_{i}(j)=r_{i}(j-1)\end{cases}
$$

Set $V_{L}(b):=0$ if $C_{i, j} \subseteq \operatorname{Im}\left(F_{N_{i-1}}\right)$, that is, if $j \leq h_{i}$. If $j>h_{i}$ then there is a unique index $\nu \in\{1, \ldots, \ell\}$ such that $j=s_{i-1}(\nu)$, in which case we set $V_{L}(b):=t_{i-1, \nu}^{-1}(b) \in B_{i-1, \nu}$. With 
these definitions, $L$ becomes a Dieudonné module of type $(d, \mathfrak{f})$; it should be thought of as the "associated graded" of $N$ with respect to the canonical filtrations $C_{i, \bullet}$

We claim that a choice of bases $\beta_{a}$ as in the lemma gives an isomorphism of $L$ with the standard Dieudonné module $N_{\underline{w}}$. To see this we have to investigate the relation between the permutations $\rho_{i}$ and the $\dot{w}_{i}$, writing $\underline{w}=\underline{w}(\mathscr{H}, \theta)=\left\{w_{i}\right\}_{i \in \mathscr{I}}$.

In order to describe this relation, we first have to "stretch" the permutations $\rho_{i}$ to become elements of $\mathfrak{S}_{d}$; the stretching factors are precisely the dimensions of the blocks $B_{i, j}$. More formally, for $i \in \mathscr{I}$, consider the subset $V_{i} \subset\{1, \ldots, \ell\} \times \mathbb{Z}_{\geq 1}$ consisting of all pairs $(j, n)$ with $n \leq \operatorname{dim}\left(B_{i, j}\right)$. Define a total ordering of this set by

$$
(j, n)<\left(j^{\prime}, n^{\prime}\right) \quad \stackrel{\text { def }}{\Leftrightarrow} \quad j<j^{\prime} \quad \text { or } \quad j=j^{\prime} \text { and } n<n^{\prime} .
$$

Write $\zeta_{i}:\{1, \ldots, d\} \stackrel{\sim}{\longrightarrow} V_{i}$ for the unique order-preserving bijection. Now "stretch" $\rho_{i}$ to a bijection $\rho_{i}^{\sharp}: V_{i} \rightarrow V_{i+1}$ by $\rho_{i}^{\sharp}(j, n)=\left(\rho_{i}(j), n\right)$. Via the bijections $\zeta_{i}$ and $\zeta_{i+1}$ we can view $\rho_{i}^{\sharp}$ as an element of $\mathfrak{S}_{d}$.

Next define $\pi_{i} \in \mathfrak{S}_{d}$ by

$$
\pi_{i}(\nu)= \begin{cases}\mathfrak{f}(i)+\nu & \text { if } \nu \leq d-\mathfrak{f}(i) \\ \nu-(d-\mathfrak{f}(i)) & \text { if } \nu>d-\mathfrak{f}(i)\end{cases}
$$

Then the permutations $\rho_{i}^{\sharp}$ and $\dot{w}_{i}$ are related by the formula $\dot{w}_{i}=\pi_{i} \circ \rho_{i}^{\sharp}$. Using this, one now easily verifies that, indeed, a choice of bases $\beta_{a}$ as in the lemma gives an isomorphism of Dieudonné modules with $\kappa$-action $L \stackrel{\sim}{\longrightarrow} N_{\underline{w}}$.

To make this concrete in an example, suppose that $\kappa$ has $p^{3}$ elements. Identify $\mathscr{I}=\mathbb{Z} / 3 \mathbb{Z}$, take $d=6$ and $\mathfrak{f}(1)=2, \mathfrak{f}(2)=1, \mathfrak{f}(3)=5$. Consider the standard object $\left(\mathscr{H}_{\underline{w}}, \theta_{\underline{w}}\right)$, with $\underline{w}$ given by

$$
\dot{w}_{1}=\left[\begin{array}{llllll}
1 & 2 & 3 & 4 & 5 & 6 \\
1 & 3 & 4 & 5 & 2 & 6
\end{array}\right] \quad \dot{w}_{2}=\left[\begin{array}{llllll}
1 & 2 & 3 & 4 & 5 & 6 \\
2 & 3 & 1 & 4 & 5 & 6
\end{array}\right] \quad \dot{w}_{3}=\left[\begin{array}{llllll}
1 & 2 & 3 & 4 & 5 & 6 \\
1 & 2 & 6 & 3 & 4 & 5
\end{array}\right] .
$$

The reader is invited to check that in this case the canonical blocks, and the way they are permuted, look as follows:

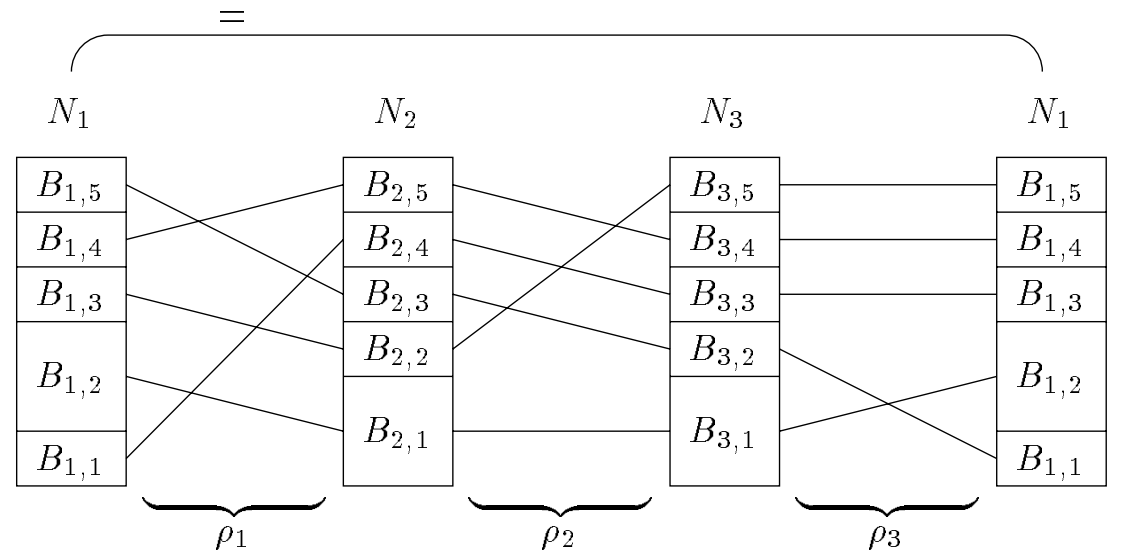

In each $N_{i}$ there are four blocks of dimension 1 and one 2-dimensional block. The permutations $\rho_{i}$ are given by

$$
\rho_{1}=\left[\begin{array}{lllll}
1 & 2 & 3 & 4 & 5 \\
4 & 1 & 2 & 5 & 3
\end{array}\right] \quad \rho_{2}=\left[\begin{array}{lllll}
1 & 2 & 3 & 4 & 5 \\
1 & 5 & 2 & 3 & 4
\end{array}\right] \quad \rho_{3}=\left[\begin{array}{llllll}
1 & 2 & 3 & 4 & 5 & 6 \\
2 & 1 & 3 & 4 & 5 &
\end{array}\right] ;
$$


their stretched versions are

$$
\rho_{1}^{\sharp}=\left[\begin{array}{llllll}
1 & 2 & 3 & 4 & 5 & 6 \\
5 & 1 & 2 & 3 & 6 & 4
\end{array}\right] \quad \rho_{2}^{\sharp}=\left[\begin{array}{llllll}
1 & 2 & 3 & 4 & 5 & 6 \\
1 & 2 & 6 & 3 & 4 & 5
\end{array}\right] \quad \rho_{3}^{\sharp}=\left[\begin{array}{llllll}
1 & 2 & 3 & 4 & 5 & \\
2 & 3 & 1 & 4 & 5 & 6
\end{array}\right] .
$$

4.12. F-V-compatible liftings. - Recall that for $a \in \mathscr{A}$, we call $t_{a}: B_{a} \rightarrow B_{\rho(a)}$ an $F$-arrow or $V$-arrow according to whether it is induced by Frobenius or (inversely) by Verschiebung.

Fix bases $\beta_{a}$ as in Lemma 4.10. We are going to lift each $\beta_{a}$ to an ordered subset $\gamma_{a} \subset C_{a}$. (Here $a=(i, j) \in \mathscr{A}$ and $C_{a}:=C_{i, j}$.) For every $i \in \mathscr{I}$ this will give us an ordered basis $\gamma_{i}$ of $N_{i}$ with the property that each $C_{i, j}$ is spanned by the first $\operatorname{dim}\left(C_{i, j}\right)$ base vectors. (To get the correct indexing for the basis $\gamma_{i}$ we use the bijections $\zeta_{i}$ of 4.11.) We want to find liftings $\gamma_{a}$ that satisfy the following two conditions:

(i) If $t_{a}$ is an $F$-arrow then $\gamma_{\rho(a)}$ is the image of $\gamma_{a}$ under $F_{N}: C_{a} \rightarrow C_{\rho(a)}$;

(ii) If $t_{a}$ is a $V$-arrow then $\gamma_{a}$ is the image of $\gamma_{\rho(a)}$ under $V_{N}: C_{a} \leftarrow C_{\rho(a)}$.

If these two conditions are satisfied we shall say that $\left\{\gamma_{a}\right\}_{a \in \mathscr{A}}$ is an $F$-V-compatible collection of liftings. If such a collection of liftings exists then it induces an isomorphism of $N$ with the Dieudonné module $L$ as in the above remark, and it follows from that remark that $(\mathscr{H}, \theta) \cong$ $\left(\mathscr{H}_{\underline{w}}, \theta_{\underline{w}}\right)$.

4.13. Tops, bottoms, and chains. - Consider three consecutive elements $a(-1), a(0)=$ $\rho(a(-1))$ and $a(1)=\rho(a(0))$. We say that $a(0) \in \mathscr{A}$ is a top if $t_{a(0)}$ is an $F$-arrow and $t_{a(-1)}$ is a $V$-arrow, i.e., if both Frobenius and Verschiebung are injective on $B_{a(0)}$. We say that $a(0)$ is a bottom if $t_{a(0)}$ is a $V$-arrow and $t_{a(-1)}$ is an $F$-arrow, i.e., if Frobenius and Verschiebung are both zero on $B_{a(0)}$.

$$
\text { top: } \stackrel{V}{\longleftarrow} \cdot \stackrel{F}{\longrightarrow} \quad \text { bottom: } \quad \stackrel{F}{\longrightarrow} \cdot \stackrel{V}{\longleftarrow}-
$$

Write $\mathscr{A}^{t} \subset \mathscr{A}$ for the set of tops, $\mathscr{A}^{b} \subset \mathscr{A}$ for the set of bottom elements.

By an $F$-chain in $\mathscr{A}$ we mean a sequence $a(0), a(1), \ldots, a(n)$ such that $a(0)$ is a top, $a(n)$ is a bottom, and each of the maps $t_{a(\nu)}(0 \leq \nu<n)$ is an $F$-arrow. Similarly, by a $V$-chain in $\mathscr{A}$ we mean a sequence $a(-n), a(-n+1), \ldots, a(0)$ such that $a(0)$ is a top, $a(-n)$ is a bottom, and each of the maps $t_{a(\nu)}(-n \leq \nu<0)$ is a $V$-arrow. With this terminology, the orbits in $\mathscr{A}$ can be pictured as follows:

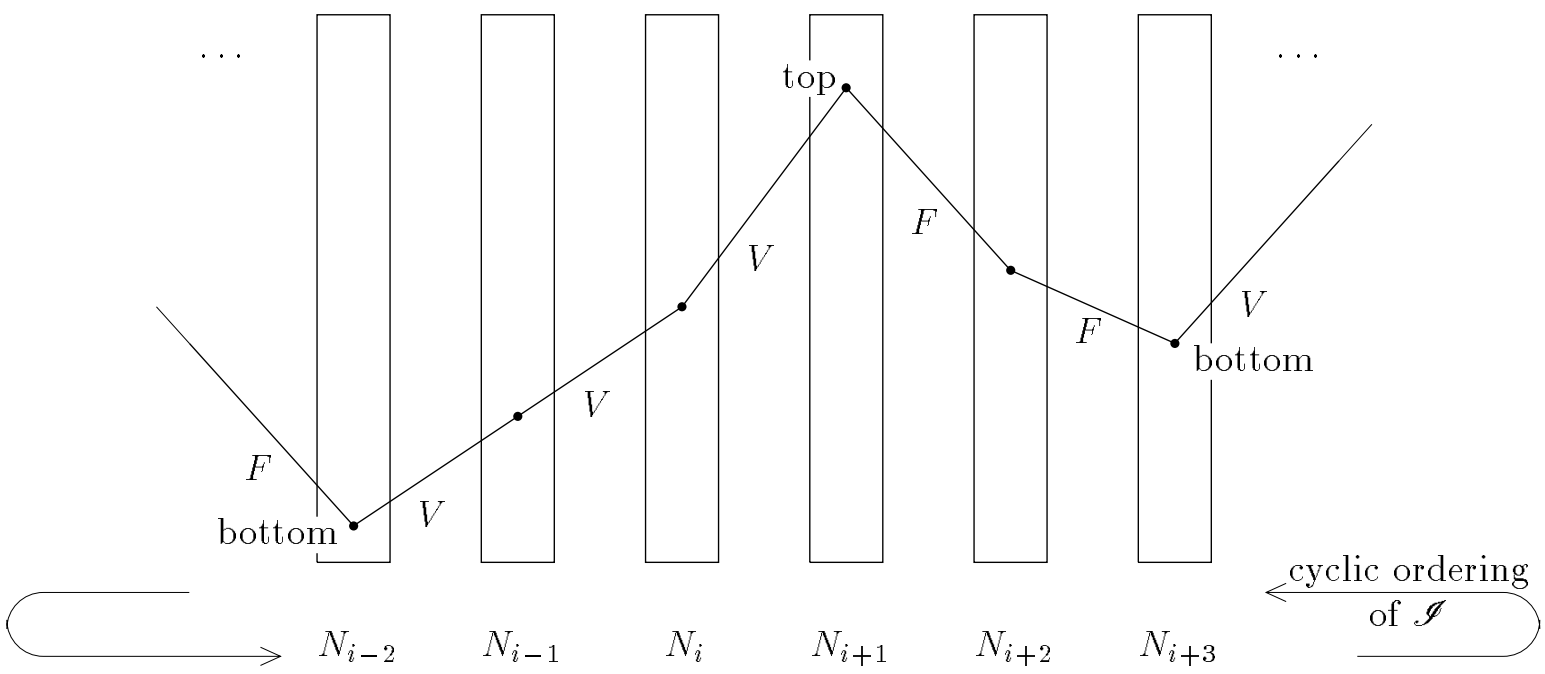

(Going round, the orbit will in general cross itself; this we have not illustrated.) 
4.14. Pairs of marked orbits. - For the arguments that follow we need some observations about the mutual position of two orbits. We begin with some terminology. Let $a=(i, j)$ and $a^{\prime}=\left(i^{\prime}, j^{\prime}\right)$ be two elements of $\mathscr{A}$. We say that $a$ and $a^{\prime}$ are comparable if $i=i^{\prime}$. We write $a^{\prime} \prec a$ if $i^{\prime}=i$ and $j^{\prime}<j$.

If $O \subseteq \mathscr{A}$ is an orbit then by a marking of $O$ we mean a map $\mathbb{Z} \rightarrow O$, written $n \mapsto a(n)$, such that $a(n+1)=\rho(a(n))$ for all $n$. Clearly such a marking is fully determined if we specify one $a(n)$. Let $n \mapsto a(n)$ and $n \mapsto a^{\prime}(n)$ be markings of orbits $O$ and $O^{\prime}$, respectively. We assume that $a(n)$ and $a^{\prime}(n)$ are comparable for all (equivalently: some) $n$. Given integers $n_{1}<n_{2}$ we say that $O$ and $O^{\prime}$ are parallel in the interval $\left[n_{1}, n_{2}\right]$ if for all $\nu$ with $n_{1} \leq \nu<n_{2}$ the two arrows $t_{a(n)}$ and $t_{a^{\prime}(n)}$ are of the same kind, i.e., they are either both $F$-arrows or both $V$-arrows. We say that the (ordered) pair of orbits $O$ and $O^{\prime}$ is up-down at index $n$ if $t_{a(n)}$ is a $V$-arrow, $t_{a^{\prime}(n)}$ is an $F$-arrow, and that they are down-up at $n$ if it is the other way around.

Suppose that $a^{\prime}(0)=\left(i_{0}^{\prime}, j_{0}^{\prime}\right) \prec a(0)=\left(i_{0}, j_{0}\right)$. Let us first follow $O$ and $O^{\prime}$ backwards in time. We know that $t_{a(-1)}: B_{a(-1)} \rightarrow B_{a(0)}$ is an $F$-arrow if and only if $j_{0} \leq h_{i_{0}}$. (This is really the definition of $h_{i_{0}}$; see 4.4.) In particular, if $t_{a(-1)}$ is an $F$-arrow then so is $t_{a^{\prime}(-1)}$, and in this case it is immediate from the definitions that also $a^{\prime}(-1) \prec a(-1)$. Similarly, if $t_{a^{\prime}(-1)}$ is a $V$-arrow then so is $t_{a(-1)}$, in which case we again have $a^{\prime}(-1) \prec a(-1)$. Thus, if $O$ and $O^{\prime}$ are parallel in the interval $\left[n_{1}, 0\right]$ and $a^{\prime}(0) \prec a(0)$ then $a^{\prime}(\nu) \prec a(\nu)$ for all $\nu \in\left[n_{1}, 0\right]$. Note that if $O$ and $O^{\prime}$ are no longer parallel in $\left[n_{1}-1,0\right]$ then we may still have that $a^{\prime}\left(n_{1}-1\right) \prec a\left(n_{1}-1\right)$. (Configurations $c$. and $\mathrm{d}$. in the illustration below.)

Still assuming that $a^{\prime}(0) \prec a(0)$, let us now follow $O$ and $O^{\prime}$ forward in time. Suppose they are parallel in the interval $\left[0, n_{2}\right]$ but not in $\left[0, n_{2}+1\right]$. We find that $a^{\prime}(\nu) \prec a(\nu)$ for all positive $\nu \leq n$. At index $n$ the pair $\left(O, O^{\prime}\right)$ may be either down-up or up-down. In the down-up case we necessarily have $a^{\prime}\left(n_{2}+1\right) \succ a\left(n_{2}+1\right)$.

To summarize, if $O$ and $O^{\prime}$ are marked orbits with $a^{\prime}(0) \prec a(0)$ then the possible configurations are

a.

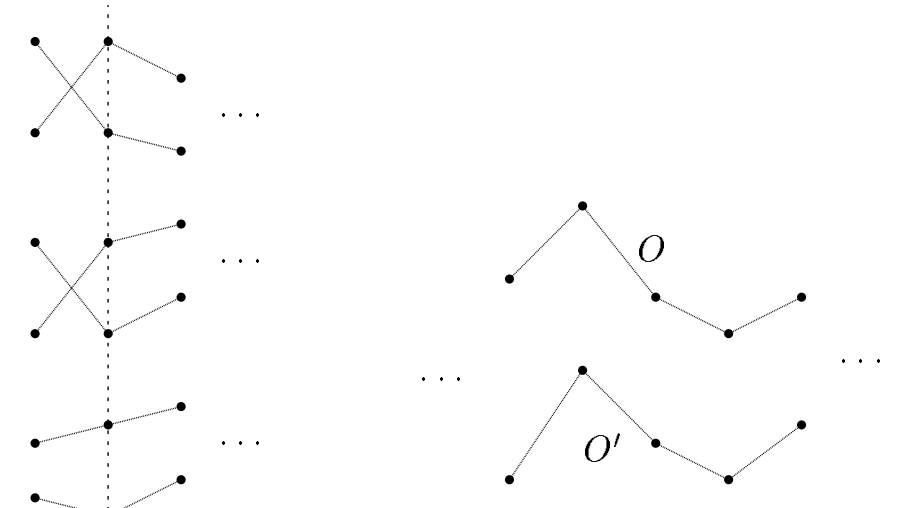

d.

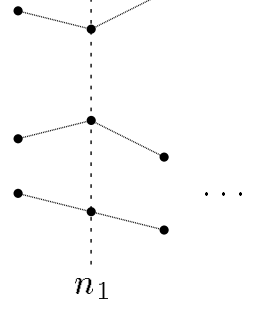

parallel

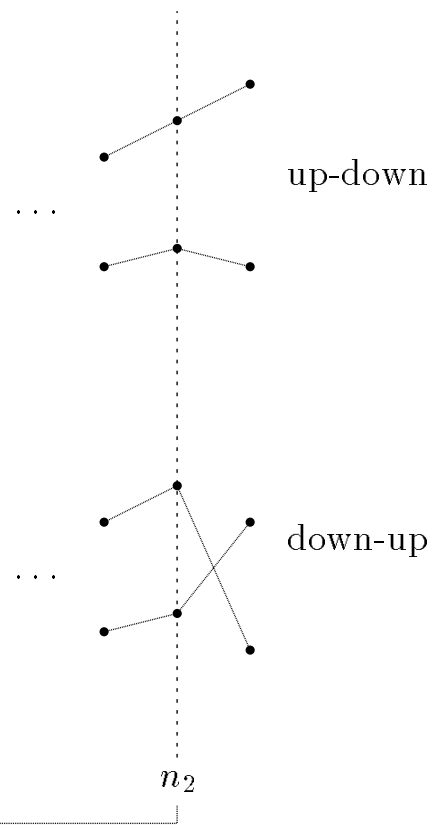

4.15. Lemma. - Let $O$ and $O^{\prime}$ be marked orbits as above.

(i) If $O \neq O^{\prime}$ (as marked orbits) then there is no infinite interval in which $O$ and $O^{\prime}$ are parallel. 
(ii) Suppose that $a^{\prime}(0) \prec a(0)$. Let $e:=\# O$ and suppose that $O$ and $O^{\prime}$ are parallel in the interval $[-e, 0]$. Then $a^{\prime}(-e) \prec a^{\prime}(0) \prec a(-e)=a(0)$.

Proof. (i) There is an integer $N$ (the l.c.m. of the lengths of $O$ and $\left.O^{\prime}\right)$ such that $a(n+N)=$ $a(n)$ and $a^{\prime}(n+N)=a^{\prime}(n)$ for all $n$. Thus, if there is an infinite interval in which $O$ and $O^{\prime}$ are parallel, then they are actually parallel in all of $\mathbb{Z}$. This implies that for every word $Z$ in the letters $F$ and $V^{-1}$ we have $C_{a(0)} \subseteq Z(N)$ if and only if $C_{a^{\prime}(0)} \subseteq Z(N)$. (See 2.5 for notations.) By definition of the filtrations $C_{i}, \bullet$ and the assumption that $a(0)$ and $a^{\prime}(0)$ are comparable, this is possible only if $a(0)=a^{\prime}(0)$. But this contradicts the assumption that $a(0) \prec a^{\prime}(0)$.

(ii) Let $\alpha=(i, j)$ and $\alpha^{\prime}=\left(i, j^{\prime}\right)$ be two comparable elements of $\mathscr{A}$. Suppose that $\rho\left(\alpha^{\prime}\right) \prec$ $\rho(\alpha)$; in other words, $\rho_{i}\left(j^{\prime}\right)<\rho_{i}(j)$. If $t_{\alpha}$ and $t_{\alpha^{\prime}}$ are both $F$-arrows or both $V$-arrows then $\left(\rho_{i}(j)-\rho_{i}\left(j^{\prime}\right)\right) \leq j-j^{\prime}$. Iterating this $e$ times we obtain (ii); here we have to remark that by (i) we cannot have $a^{\prime}(-e)=a^{\prime}(0)$.

Now we are ready to prove what we want.

4.16. Proposition. - Let $\left\{\beta_{a}\right\}_{a \in \mathscr{A}}$ be a collection of ordered bases as in 4.10. Then we can lift each $\beta_{a}$ to an ordered subset $\gamma_{a} \subset C_{a}$ such that the collection $\left\{\gamma_{a}\right\}$ is $F$-V -compatible.

As remarked in 4.12 above, if we prove the proposition then Theorem 4.7 follows.

Proof. It suffices to find $F-V$-compatible liftings $\gamma_{a}$ for $a$ running through a fixed orbit $O \subset \mathscr{A}$. In fact, by induction on the integer $r \geq 0$ we shall prove the following assertion:

$L(r):$ If $O \subset \mathscr{A}$ is an orbit and $O$ contains an element $(i, j)$ with $j \leq r$ then we can lift the $\beta_{a}$ for $a \in O$ to an $F-V$-compatible collection $\left\{\gamma_{a}\right\}_{a \in O}$.

Let us first make explicit three remarks that follow directly from the definitions:

(1) Consider an $F$-chain $a(0), \ldots, a(n)$. Let $\gamma_{a(0)}$ be a lifting of $\beta_{a(0)}$ inside $C_{a(0)}$. For $0 \leq \nu \leq n$, let $\gamma_{a(\nu)}$ be the image (as an ordered set) of $\gamma_{a(0)}$ under $F_{N}^{\nu}$. Then $\gamma_{a(\nu)}$ is a lifting of $\beta_{a(\nu)}$ inside $C_{a(\nu)}$.

(2) Consider a $V$-chain $a(-n), \ldots, a(0)$. Let $\gamma_{a(0)}$ be a lifting of $\beta_{a(0)}$ inside $C_{a(0)}$. For $0 \leq \nu \leq n$, let $\gamma_{a(-\nu)}$ be the image (as an ordered set) of $\gamma_{a(0)}$ under $V_{N}^{\nu}$. Then $\gamma_{a(-\nu)}$ is a lifting of $\beta_{a(-\nu)}$ inside $C_{a(-\nu)}$.

(3) Consider an $F$-chain $a(0), \ldots, a(n)$. Let $\gamma_{a(n)}$ be a lifting of $\beta_{a(n)}$ inside $C_{a(n)}$. Then there is a lifting $\gamma_{a(0)}$ of $\beta_{a(0)}$ inside $C_{a(0)}$ such that $\gamma_{a(n)}$ is the image of $\gamma_{a(0)}$ under $F_{N}^{n}$.

Fix an orbit $O \subset \mathscr{A}$ and a marking $\mathbb{Z} \rightarrow O$, denoted $n \mapsto a(n)$. Let $e:=\# O$. As a first approximation we shall choose, for $n$ in some subset of $\mathbb{Z}$, liftings $\Gamma(n) \subset C_{a(n)}$ of $\beta_{a(n)}$. These liftings will be compatible under $F$ and $V$, but in general $\Gamma(n+e)$ will be different from $\Gamma(n)$. After that, we shall modify the $\Gamma(n)$ such that $\Gamma(n+e)=\Gamma(n)$ for all $n$, in which case we can define the desired liftings $\gamma_{a}$ by $\gamma_{a(n)}:=\Gamma(n)$.

Suppose there are $q$ top elements and $q$ bottom elements in $O$. Let

$$
\cdots<b_{0}<t_{0}<b_{1}<t_{1}<\cdots<b_{q-1}<t_{q-1}<b_{q}=\left(b_{0}+e\right)<t_{q}=\left(t_{0}+e\right)<\cdots
$$

be the integers such that the $a\left(b_{n}\right)$ are the bottom elements and the $a\left(t_{n}\right)$ are the top elements.

Start by choosing a lifting $\Gamma\left(b_{q}\right)$ of $\beta_{a\left(b_{q}\right)}=\beta_{a\left(b_{0}\right)}$. We apply (3). This tells us that we can choose a lifting $\Gamma\left(t_{q-1}\right)$ such that $\Gamma\left(b_{q}\right)$ is the image of $\Gamma\left(t_{q-1}\right)$ under $F_{N}^{n}$, where $n=b_{q}-t_{q-1}$. By construction, the elements $a\left(t_{q-1}+\nu\right)$ with $0 \leq \nu \leq b_{q}-t_{q-1}$ form an $F$-chain. The condition that the $\Gamma(n)$ are $F-V$-compatible forces us to define $\Gamma\left(t_{q-1}+\nu\right)$ as the image of $\Gamma\left(t_{q-1}\right)$ under $F_{N}^{\nu}$. Similarly, the elements $a\left(t_{q-1}-\mu\right)$ for $0 \leq \mu \leq t_{q-1}-b_{q-1}$ form a $V$-chain, and we define $\Gamma\left(t_{q-1}-\mu\right)$ as the image of $\Gamma\left(t_{q-1}\right)$ under $V_{N}^{\mu}$. 
We repeat the previous step, now starting with $\Gamma\left(b_{q-1}\right)$. This gives us liftings $\Gamma(n)$ for $b_{q-2} \leq n \leq b_{q-1}$. We continue this process until we arrive at $b_{0}$. Notice that $\Gamma\left(b_{0}\right)$ and $\Gamma\left(b_{q}\right)$ are both liftings of $\beta_{a\left(b_{0}\right)}$ inside $C_{a\left(b_{0}\right)}$, but that in general they will be different.

Next we want to modify the $\Gamma(n)$, for $b_{0} \leq n \leq b_{q}$, to ordered sets $\Gamma^{\prime}(n)$, in such a way that the new liftings are still $F$ - $V$-compatible but also satisfy $\Gamma^{\prime}\left(b_{0}\right)=\Gamma^{\prime}\left(b_{q}\right)$. Write $a\left(b_{0}\right)=a\left(b_{q}\right)=(i, j)$. We may assume that $b_{0}$ was chosen such that $j$ is minimal among the second coefficients of the elements $a \in O$. By induction we may further assume that statement $L(j-1)$ is already proven. Here we should remark that for $j=1$ there is nothing left to prove, as in this case we have $B_{a\left(b_{0}\right)}=C_{a\left(b_{0}\right)}$ and $\Gamma\left(b_{0}\right)=\beta_{a\left(b_{0}\right)}=\Gamma\left(b_{q}\right)$.

Set $\delta\left(b_{q}\right):=\Gamma\left(b_{q}\right)-\Gamma\left(b_{0}\right)$. (Concretely: if $\Gamma\left(b_{q}\right)=x_{1}, \ldots, x_{d}$ and $\Gamma\left(b_{0}\right)=y_{1}, \ldots, y_{d}$, with $d=\operatorname{dim}\left(B_{i, j}\right)$, then $\delta\left(b_{q}\right)$ is the ordered set $\left.x_{1}-y_{1}, \ldots, x_{d}-y_{d}.\right)$ As $\Gamma\left(b_{q}\right)$ and $\Gamma\left(b_{0}\right)$ are both liftings of $\beta_{a\left(b_{0}\right)}$ we have $\delta\left(b_{q}\right) \subset C_{i, j-1}$. If $\Gamma\left(b_{q}\right)=\Gamma\left(b_{0}\right)$ we are done. If not, let $J$ (with $J \leq j-1$ ) be the largest index such that $\delta\left(b_{q}\right)$ is not contained in $C_{i, J-1}$. By downward induction on $J$ it suffices to show that we can modify the $\Gamma(n)$ to $F$ - $V$-compatible liftings $\Gamma^{\prime}(n)$ such that $\Gamma^{\prime}\left(b_{q}\right)-\Gamma^{\prime}\left(b_{0}\right) \subset C_{i, J-1}$.

Let $O^{\prime}$ be the orbit of $(i, J)$. Let $a^{\prime}: \mathbb{Z} \rightarrow O^{\prime}$ be the marking with $a^{\prime}\left(b_{q}\right)=(i, J)$. We now apply the induction hypothesis $L(j-1)$. It follows that for $b_{0} \leq n \leq b_{q}$ we can find ordered sets $\varepsilon(n) \subset C_{a^{\prime}(n)}$, of cardinality $d=\operatorname{dim}\left(B_{i, j}\right)$, in such a way that

(a) the $\varepsilon(n)$ are $F$ - $V$-compatible (with respect to the orbit $O^{\prime}$ );

(b) $\varepsilon\left(b_{q}\right)$ and $\delta\left(b_{q}\right)$ have the same image in $B_{i, J}$.

To conclude the proof, there are two cases to consider. First assume that the marked orbits $O$ and $O^{\prime}$ are not parallel in the interval $\left[b_{0}, b_{q}\right]$. Let $\nu \in\left[b_{0}, b_{q}\right]$ be the largest index such that $t_{a^{\prime}(\nu)}$ and $t_{a(\nu)}$ are of the opposite kind. For $b_{0} \leq n \leq b_{q}$ define $\Gamma^{\prime}(n)$ by

$$
\Gamma^{\prime}(n)= \begin{cases}\Gamma(n) & \text { if } n \leq \nu \\ \Gamma(n)-\varepsilon(n) & \text { if } n>\nu\end{cases}
$$

Now the whole point is that, because $t_{a^{\prime}(\nu)}$ and $t_{a(\nu)}$ are of the opposite kind, these $\Gamma^{\prime}(n)$ are again $F-V$-compatible. By construction, $\Gamma^{\prime}\left(b_{q}\right)-\Gamma^{\prime}\left(b_{0}\right)=\Gamma\left(b_{q}\right)-\Gamma\left(b_{0}\right)-\varepsilon\left(b_{q}\right)$ is contained in $C_{i, J-1}$.

The other possibility is that $O$ and $O^{\prime}$ are parallel in the interval $\left[b_{0}, b_{q}\right]$. In this case, set

$$
\Gamma^{\prime}(n):=\Gamma(n)-\varepsilon(n) .
$$

These are again $F$-V-compatible, and it follows from (ii) of Lemma 4.15 that also in this case $\Gamma^{\prime}\left(b_{q}\right)-\Gamma^{\prime}\left(b_{0}\right)$ is contained in $C_{i, J-1}$. Proceeding by induction this finally gives us the desired liftings $\gamma_{a}$.

4.17. To conclude this section, let us draw a conclusion from Theorem 4.7 that will be of use in the next section.

We say that a pair $(\mathscr{H}, \theta)$ is indecomposable if it is not isomorphic to the product of two non-trivial $\mathrm{BT}_{1}$ 's with $\kappa$-action, and that it is isotypic if it is isomorphic to a power of an indecomposable pair. Viewing the Dieudonné module $N$ as a module over the (non-commutative) ring $\Lambda_{\kappa}:=\kappa \otimes_{\mathbb{F}_{p}} k_{\sigma}[F, V] /(F V, V F)$, these notions correspond to the usual notions for $\Lambda_{\kappa^{-}}$ modules. Notice that $N$ is both noetherian and artinian as a $\Lambda_{\kappa}$-module, since it has finite $k$ dimension. In particular, $N$ admits a unique decomposition as a direct sum of indecomposables. For the pair $(\mathscr{H}, \theta)$ this means that it can be written as a product of indecomposable pairs, and that the factors in such a decomposition are uniquely determined. The same conclusions can 
also be derived from the theorem and its proof; in fact, we see that the isotypic factors can be read from the decomposition of the set $\mathscr{A}$ into orbits:

4.18. Corollary. - The pair $(\mathscr{H}, \theta)$ is isotypic if and only if the associated index set $\mathscr{A}$ consists of a single orbit. In general, the decomposition of $\mathscr{A}$ into orbits corresponds to a decomposition of $(\mathscr{H}, \theta)$ as a product of isotypic pairs. The pair $(\mathscr{H}, \theta)$ is indecomposable if and only if $\mathscr{A}$ consists of a single orbit and all canonical blocks are 1-dimensional.

For instance, the pair $(\mathscr{H}, \theta)$ corresponding to the example in 4.11 decomposes as a product of three isotypic factors. Two of the factors are indecomposable; the third one is the square of an indecomposable pair.

\section{5. $\mathrm{BT}_{1}$ 's with endomorphisms and a polarization - cases $\mathrm{C}$ and $\mathrm{D}$}

In this section we state, and solve, a problem (GPE) about $\mathrm{BT}_{1}$ 's with an action of a semisimple $\mathbb{F}_{p}$-algebra and a pq-polarization. After some reduction steps the main result is given in Theorem 5.5. The problem splits up in three cases, referred to as (C), (D) and (A). Mainly for notational reasons we shall deal with case $(\mathrm{A})$ in the next section.

5.1. The setup. - Let $k$ be an algebraically closed field of characteristic $p>0$. Let $(D, *)$ be a finite dimensional semi-simple $\mathbb{F}_{p}$-algebra with involution. If $(\mathscr{G}, \lambda)$ is a pq-polarized $\mathrm{BT}_{1}$ over $k$, write $f \mapsto f^{\dagger}$ for the Rosati involution on $\operatorname{End}_{k}(\mathscr{G})$ induced by $\lambda$. The problem that we want to consider in this section is:

(GPE) Classification of triples $(\mathscr{G}, \lambda, \iota)$, where $(\mathscr{G}, \lambda)$ is a pq-polarized $\mathrm{BT}_{1}$ over $k$ and $\iota: D \rightarrow$ $\operatorname{End}_{k}(\mathscr{G})$ is a homomorphism with $\iota(1)=\mathrm{id}_{\mathscr{G}}$ and $\iota\left(d^{*}\right)=\iota(d)^{\dagger}$ for all $d \in D$.

This problem is easily reduced to the case that $(D, *)$ is a simple algebra with involution. Namely, suppose we have a decomposition of $(D, *)$ as a product of algebras with involution $\left(D_{i}, *_{i}\right)$. Choose idempotents $e_{i} \in D$ with $D_{i}=e_{i} \cdot D$. Then $\mathscr{G}_{i}:=\operatorname{Im}\left(\iota\left(e_{i}\right): \mathscr{G} \rightarrow \mathscr{G}\right)$ is again a $\mathrm{BT}_{1}$, the pq-polarization $\lambda$ induces a pq-polarization $\lambda_{i}$ on $\mathscr{G}_{i}$, and $(\mathscr{G}, \lambda)$ is the product of the $\left(\mathscr{G}_{i}, \lambda_{i}\right)$ thus obtained.

From now on we assume that $(D, *)$ is simple as an algebra with involution. Write $\tilde{\kappa}$ for the center of $D$ and put $\kappa=\left\{a \in \tilde{\kappa} \mid a^{*}=a\right\}$. Then $\kappa$ is a finite field and we can distinguish the following possibilities:

(C) the involution $*$ is orthogonal (of the first kind) and $\tilde{\kappa}=\kappa$;

(D) the involution $*$ is symplectic (of the first kind) and $\tilde{\kappa}=\kappa$;

(A) the involution $*$ is of the second kind; either $\tilde{\kappa} \cong \kappa \times \kappa$ (case (A1)) or $\tilde{\kappa}$ is a quadratic extension of $\kappa$ (case (A2)).

(The chosen labels refer to the root systems of the algebraic groups that will come into play.) If char $(k)=2$ then we shall exclude the case that $*$ is a symplectic involution. In this section we shall only consider cases (C) and (D). The structures we find in case (A) are not very different but require additional notations; we shall therefore discuss this case in a separate section. Throughout, we freely use the notations and terminology introduced in the previous section.

5.2. Morita equivalence. - By Dieudonné theory, the category of triples $(\mathscr{G}, \lambda, \iota)$ as in (GPE) is equivalent to the category $\mathbf{C}_{1}$ of 5 -tuples $\left(M, F_{M}, V_{M}, \varphi, \iota\right)$, where

- $\left(M, F_{M}, V_{N}\right)$ is the Dieudonné module of a $\mathrm{BT}_{1}$;

- $\varphi: M \times M \rightarrow k$ is a symplectic form with $\varphi\left(F m_{1}, m_{2}\right)=\varphi\left(m_{1}, V m_{2}\right)^{p}$ for all $m_{1}$, $m_{2} \in M$ 
- $\iota: D \rightarrow \operatorname{End}_{\mathrm{DM}}(M)$ defines an action of $D$ on the Dieudonné module $M$ such that $\varphi\left(d m_{1}, m_{2}\right)=\varphi\left(m_{1}, d^{*} m_{2}\right)$ for all $d \in D$ and $m_{1}, m_{2} \in M$.

Recall from 4.3 that $M$ is free as a $K$-module.

We have a Morita equivalence, which tells us that the category $\mathbf{C}_{1}$ is equivalent to a category $\mathbf{C}_{2}$ whose objects are of a simpler kind. The precise description of the category $\mathbf{C}_{2}$ depends on the type of $(D, *)$. Namely, the objects involve pairs $(N, \Psi)$, where $N$ is a free $K$-module of finite rank, and where $\Psi: N \times N \rightarrow K$ is

(C) a symplectic form if $*$ is orthogonal;

(D) an orthogonal form if $*$ is symplectic $(\operatorname{char}(k) \neq 2)$.

(We shall see that the $K$-rank of $N$ is necessarily even; this explains why the orthogonal case is labelled (D) and a type (B) does not occur.) Now define $\mathrm{C}_{2}$ as the category of 5-tuples $\left(N, F_{N}, V_{N}, \Psi, \theta\right)$, where

- $\left(N, F_{N}, V_{N}\right)$ is the Dieudonné module of a $\mathrm{BT}_{1}$;

- $\theta: \kappa \rightarrow \operatorname{End}_{\mathrm{DM}}(N)$ defines an action of $\kappa$ on the Dieudonné module $N$; via this action we can view $N$ as a module over $K$, and we require that it is free;

- $\Psi: N \times N \rightarrow K$ is a form as just described, such that $\Psi\left(F n_{1}, n_{2}\right)=F_{K}\left(\Psi\left(n_{1}, V n_{2}\right)\right)$ for all $n_{1}, n_{2} \in N$.

To describe the equivalence $\mathbf{C}_{2} \stackrel{\text { eq. }}{\longrightarrow} \mathbf{C}_{1}$, fix an isomorphism $D \cong M_{r}(\kappa)$ and set $\mathscr{L}:=\kappa^{r}$, viewed as a left $D$-module. The involution $*$ on $D$ corresponds to a non-degenerate form $\chi$ on $\mathscr{L}$. More precisely, there is a non-degenerate bilinear form $\chi: \mathscr{L} \times \mathscr{L} \rightarrow \kappa$, unique up to a scalar in $\kappa^{\times}$, such that

$$
\chi\left(d \cdot l_{1}, l_{2}\right)=\chi\left(l_{1}, d^{*} \cdot l_{2}\right) \quad \text { for all } d \in D \text { and } l_{1}, l_{2} \in \mathscr{L} \text {. }
$$

The form $\chi$ is alternating if $*$ is symplectic and symmetric, non-alternating if $*$ is an orthogonal involution. The equivalence $\mathbf{C}_{2} \stackrel{\text { eq. }}{\longrightarrow} \mathbf{C}_{1}$ is obtained by associating to an object $\left(N, F_{N}, V_{N}, \Psi, \theta\right)$ of $\mathrm{C}_{2}$ the 5-tuple $\left(M, F_{M}, V_{M}, \varphi, \iota\right)$ with $M:=\mathscr{L} \otimes_{\kappa} N$, with $\varphi: M \times M \rightarrow k$ the symplectic form given by

$$
\varphi\left(l_{1} \otimes n_{1}, l_{2} \otimes n_{2}\right)=\operatorname{tr}_{K / k}\left(\chi\left(l_{1}, l_{2}\right) \cdot \Psi\left(n_{1}, n_{2}\right)\right)
$$

with $D$ acting on $M$ through its natural action on $\mathscr{L}$, and with $F_{M}:=\mathrm{id} \mathscr{L} \otimes F_{N}$ and $V_{M}:=$ id $\mathscr{L} \otimes V_{N}$. That this indeed gives an equivalence of categories is more or less standard; see for instance Knus's book [4], Chap. I, especially Prop. 7.2.4 and Thm. 9.3.5. As remarked above, the $M$ are free $K$-modules; they therefore indeed correspond to free $K$-modules $N$.

Now we translate back to group schemes. Throughout we shall use $\varepsilon \in\{ \pm 1\}$ for the sign of the pairings $\Psi$ involved, i.e., $\varepsilon=-1$ in case $(\mathrm{C}), \varepsilon=1$ in case (D). Let $\mathscr{H}$ be a $\mathrm{BT}_{1}$ equipped with an action of $\kappa$. Consider isomorphisms $\mu: \mathscr{H} \stackrel{\sim}{\longrightarrow} \mathscr{H}^{D}$ commuting with the $\kappa$-actions and such that the corresponding bilinear pairing $\Psi$ on the Dieudonne module is of the type described above; we shall refer to such an isomorphism $\mu$ as a $\kappa$ - $\varepsilon$-duality. (As explained in 2.6, the precise condition we want to impose has to be phrased in terms of the pairing $\Psi$.)

In conclusion, we have reduced problem (GPE) to:

(GPE') Classification of triples $(\mathscr{H}, \mu, \theta)$, where $(\mathscr{H}, \theta)$ is a $\mathrm{BT}_{1}$ with an action of $\kappa$, and where $\mu: \mathscr{H} \stackrel{\sim}{\longrightarrow} \mathscr{H}^{D}$ is a $\kappa$ - $\varepsilon$-duality.

5.3. Decomposition into character spaces. - Let $\left(N, F_{N}, V_{N}, \Psi, \theta\right)$ be an object of the category $\mathrm{C}_{2}$. As in 4.3 , the action of $\kappa$ on $N$ gives rise to a decomposition $N=\oplus_{i \in \mathscr{I}} N_{i}$. The 
form $\Psi$ decomposes as a product $\Psi=\prod_{i \in \mathscr{I}} \psi_{i}$, where $\psi_{i}: N_{i} \times N_{i} \rightarrow k_{i}$ is of the same type as $\Psi$, and

$$
\psi_{i+1}\left(F_{N_{i}}\left(n_{1}\right), n_{2}\right)=F_{k_{i}}\left(\psi_{i}\left(n_{1}, V_{N_{i}}\left(n_{2}\right)\right)\right) \quad \text { for all } n_{1} \in N_{i} \text { and } n_{2} \in N_{i+1} \text {. }
$$

If $H_{i} \subset N_{i}$ is a $k_{i}$-submodule then

$$
F_{N_{i-1}}^{-1}\left(H_{i}^{\perp_{i}}\right)=\left(V_{N_{i-1}}\left(H_{i}\right)\right)^{\perp_{i-1}} \quad \text { and } \quad V_{N_{i}}^{-1}\left(H_{i}^{\perp_{i}}\right)=\left(F_{N_{i}}\left(H_{i}\right)\right)^{\perp_{i+1}}
$$

where $\perp_{i}$ denotes the perpendicular with respect to the form $\psi_{i}$. (In the sequel we shall simply write $\perp$ for $\perp_{i}$. We trust it will always be clear from the context what is meant.)

Combining (5.3.1) with the fact that $N_{i}[F]=\operatorname{Im}\left(V_{N_{i+1}}\right)$ and $N_{i}[V]=\operatorname{Im}\left(F_{N_{i-1}}\right)$ we see that $N_{i}[F]=N_{i}[F]^{\perp}$ and $N_{i}[V]=N_{i}[V]^{\perp}$ are maximal totally isotropic subspaces. In particular, the common dimension of the spaces $N_{i}$ is even, say $\operatorname{dim}\left(N_{i}\right)=2 q$, and $\operatorname{dim}\left(N_{i}[F]\right)=q$. Repeated further application of (5.3.1) then shows that the canonical filtration $C_{i}, \bullet$ is self-dual with respect to $\psi_{i}$. In particular, the common length $\ell$ of these filtrations is even, say $\ell=2 \mathrm{~m}$. We have $C_{i, j}^{\perp}=C_{i, 2 m-j}$. In particular, if $a=(i, j)$ and $\tilde{a}=(i, 2 m+1-j)$ then $\psi_{i}$ induces a perfect pairing

$$
\psi_{(i, j)}=\psi_{a}: B_{a} \times B_{\tilde{a}} \longrightarrow k_{i} .
$$

Summing up, the discrete invariants $(d, \mathfrak{f})$ introduced in 4.6 are given by $d=2 q$ and $\mathfrak{f}(i)=q$ for all $i$. In case (D) there is another discrete invariant that we need to keep track of. Namely, define a function

$$
\delta: \mathscr{I} \rightarrow(\mathbb{Z} / 2 \mathbb{Z}) \quad \text { by } \quad \delta(i)=\left[\operatorname{dim}_{k}\left(N_{i}[F] / N_{i}[F] \cap N_{i}[V]\right)\right] .
$$

This invariant should be thought of as a "mod $p$ " version of the crystalline discriminant, cf. [11], section 3 .

5.4. Definition of the element $\underline{w}(\mathscr{H}, \mu, \theta)$. - Let $(\mathscr{H}, \mu, \theta)$ be a triple as in (GPE'). Write $\left(N, F_{N}, V_{N}, \Psi, \theta\right)$ for the corresponding Dieudonné module. Let $2 q$ be the $K$-rank of $N$. As explained above, $(\mathscr{H}, \theta)$ is of type $(2 q, \mathfrak{f})$ where $f$ is the constant function $q$.

Let $\Phi: K^{2 q} \times K^{2 q} \rightarrow K$ and $\varphi_{i}: k_{i}^{2 q} \times k_{i}^{2 q} \rightarrow k_{i}$ be the standard symplectic/orthogonal pairings. Write $G=\operatorname{Aut}_{K}\left(K^{2 q}, \Phi\right)=\prod_{i \in \mathscr{I}} G_{i}$, with $G_{i}=\operatorname{Aut}_{k_{i}}\left(k_{i}^{2 q}, \varphi_{i}\right)$. Choose an isometry $\xi:(N, \Psi) \stackrel{\sim}{\longrightarrow}\left(K^{2 q}, \Phi\right)$; this allows us to view the $N[F]_{i}$ and $C_{i} \bullet$ as partial symplectic/orthogonal flags in $k_{i}^{2 q}$. Consider the building $\Delta:=\operatorname{Flag}_{K}\left(K^{2 q}, \Phi\right)$ of partial symplectic/orthogonal flags of $K$-modules in $K^{2 q}$; it is the join of the buildings $\Delta_{i}:=F \operatorname{lag}\left(k_{i}^{2 q}, \varphi_{i}\right)$. Let $X$, resp. $X_{i}$, be the type of $N[F] \subset K^{2 q}$, resp. $N[F]_{i} \subset k_{i}^{2 q}$. We shall write $\mathscr{W}(G ; X)$ for $\mathscr{W}(\Delta ; X)$ and $\mathscr{W}\left(G_{i} ; X_{i}\right)$ for $\mathscr{W}\left(\Delta_{i} ; X_{i}\right)$. (Even though in the orthogonal case $\Delta$ is not the building of parabolic subgroups of $G$ this should cause no confusion, as it is still true that $W_{\Delta}=W_{G}$.)

The definition of $\underline{w}(\mathscr{H}, \mu, \theta)$ now proceeds exactly as in 4.6. Namely, by (ii) of Lemma 4.5 together with what was found in Examples 3.6 and 3.9, each $C_{i}$, is in optimal position with respect to $N[F]_{i}$. This gives us a well-defined element $w^{\sharp}\left(N[F]_{i}, C_{i} \bullet \bullet\right) \in \mathscr{W}\left(G_{i} ; X_{i}\right)$. We define

$$
\underline{w}(\mathscr{H}, \mu, \theta) \in \mathscr{W}(G ; X)=\prod_{i \in \mathscr{I}} \mathscr{W}\left(G_{i} ; X_{i}\right)
$$

to be the element with $w_{i}(\mathscr{H}, \mu, \theta)=w^{\sharp}\left(N[F]_{i}, C_{i}, \bullet\right)$. This element is independent of the choice of $\xi$. It is clear from these definitions that isomorphic triples give the same element $\underline{w}$. 
As explained in 3.10, if $X_{f}$ is the type of the stabilizer of $N[F]$ in the group $\mathrm{GL}_{2 q, K}$ then there is a natural map

$$
\mathscr{W}(G ; X) \longleftrightarrow \mathscr{W}\left(\mathrm{GL}_{2 q, K} ; X_{\mathrm{f}}\right)
$$

and this maps $\underline{w}(\mathscr{H}, \mu, \theta)$ to $\underline{w}(\mathscr{H}, \theta)$.

5.5. Theorem. - Notations as above. Assume that $\operatorname{char}(k) \neq 2$. Recall that we set $\varepsilon=-1$ in case $(\mathrm{C}), \varepsilon=1$ in case (D).

(i) Let $(\mathscr{H}, \theta)$ be a $B T_{1}$ with an action of $\kappa$, of type $(2 q, \mathfrak{f}=q)$. Let $\underline{w}(\mathscr{H}, \theta)$ be the associated element of $\mathscr{W}\left(\mathrm{GL}_{2 q, K} ; X_{\mathrm{f}}\right)$. Then there exists a $\kappa$-E-duality $\mu: \mathscr{H} \rightarrow \overline{\mathscr{H}}^{D}$ if and only if $\underline{w}(\mathscr{H}, \theta)$ is in the image of the map (5.4.1).

(ii) If a duality $\mu$ as in (i) exists then it is unique up to isomorphism. In other words: sending a triple $(\mathscr{H}, \mu, \theta)$ as in (GPE') to the element $\underline{w}(\mathscr{H}, \mu, \theta) \in \mathscr{W}(G ; X)$ gives a bijection

$$
\left\{\begin{array}{c}
\text { isomorphism classes of triples } \\
(\mathscr{H}, \mu, \theta) \text { of type }(2 q, \mathfrak{f}=q)
\end{array}\right\} \rightarrow \sim \mathscr{W}(G ; X) \cong \prod_{i \in \mathscr{I}} \mathfrak{S}_{q} \backslash \mathbb{H}_{q}
$$

The "only if" statement of (i) is an immediate consequence of what was explained above. Conversely, if $\underline{w}$ is an element in the image of the map (5.4.1) then we shall see in 5.7 below that there exists a pairing of the desired kind on the Dieudonne module of the standard object $\left(\mathscr{H}_{\underline{w}}, \theta_{\underline{w}}\right)$. The proof of (ii) is more involved, and will take up a large part of the rest of this section. The proof that we give only works for $\operatorname{char}(k) \neq 2$. In case $(C)$ the theorem is still true for $\operatorname{char}(k)=2$. Our proof of this is much more involved; for the special case that $\tilde{\kappa}=\mathbb{F}_{p}$ (no non-trivial endomorphisms) see [12], Section 7.

5.6. Variant. - In case (D) we have a variant of the theorem, where we replace $G$ by its identity component; this works provided that we keep the invariant $\delta$ fixed.

Concretely, fix $q$, and set $G^{0}:=\mathrm{SO}_{2 q, K}=\prod_{i \in \mathscr{I}} G_{i}^{0}$. If $L \subset K^{2 q}$ is a maximal isotropic subspace then $\operatorname{Stab}(L) \subset G^{0}$ is a maximal parabolic subgroup. The set of all parabolics obtained in this way consists of two $G^{0}$-conjugacy classes. Let $X^{0}$ be one of these two. Now let $(\mathscr{H}, \mu, \theta)$ be a triple as in (GPE'), of type $(2 q, \mathfrak{f}=q)$. Similar to what we did in 5.4 above, we can associate to this triple an element $\underline{w}^{0}(\mathscr{H}, \mu, \theta) \in \mathscr{W}\left(G^{0} ; X^{0}\right)$. For this, choose an isometry $\xi:(N, \Psi) \stackrel{\sim}{\longrightarrow}\left(K^{2 q}, \Phi\right)$ such that the stabilizer of $N[F]$ is in the chosen class $X^{0}$. Let $P_{i}:=$ $\operatorname{Stab}\left(N[F]_{i}\right)$ and $Q_{i}:=\operatorname{Stab}\left(C_{i, \bullet}\right) \subset G_{i}^{0}$. Then $Q_{i}$ is in optimal position with respect to $P_{i}$ and we can define $\underline{w}^{0}(\mathscr{H}, \mu, \theta)$ to be the element with $w_{i}^{0}(\mathscr{H}, \mu, \theta)=w^{\sharp}\left(P_{i}, Q_{i}\right)$.

On the other hand, we have associated to $(\mathscr{H}, \mu, \theta)$ the function $\delta$. As explained in 3.9 , this invariant allows us to compute $\underline{w}^{0}(\mathscr{H}, \mu, \theta)$ from $\underline{w}(\mathscr{H}, \mu, \theta)$ and vice versa. Using this, (ii) of the theorem can be rephrased as follows:

(ii') Assume we are in case (D). Fix a function $\delta: \mathscr{I} \rightarrow(\mathbb{Z} / 2 \mathbb{Z})$. Then sending a triple $(\mathscr{H}, \mu, \theta)$ as in $(\mathrm{GPE})$ to the element $\underline{w}^{0}(\mathscr{H}, \mu, \theta)$ gives a bijection

$$
\left\{\begin{array}{l}
\text { isomorphism classes of triples } \\
(\mathscr{H}, \mu, \theta) \text { of type }(2 q, \mathfrak{f}=q, \delta)
\end{array}\right\} \stackrel{\sim}{\longrightarrow} \mathscr{W}\left(G^{0} ; X^{0}\right)
$$

This is the phrasing that will be used in our applications to Shimura varieties of PEL-type.

5.7. Pairings on standard objects. - We use the notations of 5.4. Let $\underline{w}$ be an element of $\mathscr{W}(G ; X)$. In 4.8 we have associated to $\underline{w}$ a standard Dieudonné module $N_{\underline{w}}$ with an action of $\kappa$. The underlying $K$-module is just $K^{2 q}=\oplus_{i \in \mathscr{I}} k_{i}^{2 q}$. We claim that there exists a $\kappa$ - $\varepsilon$-duality 
$\mu: \mathscr{H} \stackrel{\sim}{\longrightarrow} \mathscr{H}^{D}$, corresponding to a form $\Psi$ on the Dieudonné module, such that $\left(\mathscr{H}_{\underline{w}}, \mu, \theta_{\underline{w}}\right)$ is a triple as in (GPE'). Since the image of $\underline{w}\left(\mathscr{H}_{\underline{w}}, \mu, \theta_{\underline{w}}\right)$ under the map (5.4.1) is just $\underline{\underline{w}}\left(\mathscr{H}_{\underline{w}}, \theta_{\underline{w}}\right)$ we have $\underline{w}\left(\mathscr{H}_{\underline{w}}, \mu, \theta_{\underline{w}}\right)=\underline{w}$ for any such $\mu$.

To prove our claim, let us first recall the structure of $N_{\underline{u}}$; the description we give will also be used later. Let $\ell=2 \mathrm{~m}$ be the common length of the canonical filtrations $C_{i},$. By definition, $\mathscr{A}=\mathscr{I} \times\{1,2, \ldots, 2 m\}$. We have the permutation $\rho: \mathscr{A} \rightarrow \mathscr{A}$ which sends $(i, j)$ to $\left(i, \rho_{i}(j)\right)$. If $d_{a}:=\operatorname{dim}\left(B_{a}\right)$ then we can compute the data $\left(\rho,\left\{d_{a}\right\}_{a \in \mathscr{A}}\right)$ from $\underline{w}$ and vice versa. The $K$-module $N=N_{\underline{w}}$ is given by $N=\oplus_{a \in \mathscr{A}} B_{a}$ with $x \in \kappa$ acting on $B_{(i, j)}$ as multiplication by $i(x) \in k$. For each $a \in \mathscr{A}$ we have an ordered basis $\beta_{a}$ of $B_{a}$. Frobenius and Verschiebung are given, writing $a=(i, j)$ by

$$
F\left(\beta_{a}\right)=\left\{\begin{array}{ll}
\beta_{\rho(a)} & \text { if } \rho_{i}(j) \leq m ; \\
0 & \text { if } \rho_{i}(j)>m
\end{array} \text { and } \quad V\left(\beta_{a}\right)= \begin{cases}\beta_{\rho^{-1}(a)} & \text { if } j>m \\
0 & \text { if } j \leq m\end{cases}\right.
$$

Given $a=(i, j) \in \mathscr{A}$, we write $\tilde{a}:=(i, 2 m+1-j)$, and we call this the opposite of the element $a$. Notice that the permutation $\rho$ has the property that $\rho(\tilde{a})=\widetilde{\rho(a)}$. (Drawing an illustration as in 4.11 this means that the whole picture is symmetric under reflection in a central horizontal axis.)

Assume we are in case $(\mathrm{C})$. In order to describe the desired pairing $\Psi$, we have to introduce certain constants. If $O$ is an orbit which is not self-dual, set $c(a):=1$ for all $a \in O$. If $O$ is self-dual then it has even length, say $2 s$. Then Frob ${ }^{s}: c \mapsto c^{p^{s}}$ is the unique automorphism of the field $\mathbb{F}_{p^{2 s}}$ over $\mathbb{F}_{p^{s}}$. Write $\mathbb{F}_{p^{2 s}}^{-} \subset \mathbb{F}_{p^{2 s}}$ for the subset of elements $c$ with $c^{p^{s}}=-c$. Then we choose for every $a \in O$ a constant $c(a) \in \mathbb{F}_{p^{2 s}}^{-}$in such a way that $c(\rho(a))=c(a)^{p}$. In particular, $c(\tilde{a})=-c(a)$.

For $a \in \mathscr{A}$, let now $\psi_{a}: B_{a} \times B_{\tilde{a}} \rightarrow k$ be the perfect bilinear pairing given on the ordered bases $\beta_{a}$ and $\beta_{\tilde{a}}$ by the matrix

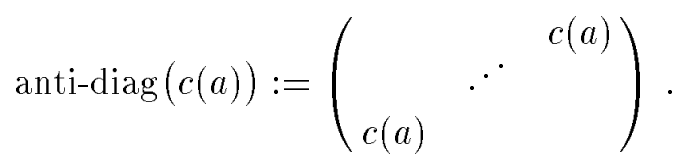

For $i \in \mathscr{I}$, let $\psi_{i}: N_{i} \times N_{i} \rightarrow k_{i}$ be the orthogonal sum of the pairings $\psi_{(i, j)}$; one checks that this is indeed a symplectic pairing. Further, the construction is such that the form $\Psi:=$ $\prod_{i \in \mathscr{I}} \psi_{i}: N \times N \rightarrow K$ satisfies

$$
\Psi\left(F n_{1}, n_{2}\right)=F_{K}\left(\Psi\left(n_{1}, V n_{2}\right)\right)
$$

for all $n_{1}, n_{2} \in N$.

Next assume we are in case (D). In this case, set $c(a)=1$ for all $a \in \mathscr{A}$. The resulting form $\Psi$ is simply the standard orthogonal form on $N \cong K^{2 q}$. Again one checks that this is a form as required.

In sum, we have shown that every element $\underline{w} \in \mathscr{W}(G ; X)$ is obtained as $\underline{w}(\mathscr{H}, \mu, \theta)$ for some triple $(\mathscr{H}, \mu, \theta)$ as in (GPE').

5.8. Brief overview of the proof. - It is clear from the definitions that isomorphic triples $(\mathscr{H}, \mu, \theta)$ give the same element $\underline{w}(\mathscr{H}, \mu, \theta)$. On the other hand, we have just shown that every element of $\mathscr{W}(G ; X)$ comes froms some triple $(\mathscr{H}, \mu, \theta)$. Thus, the task that remains is to prove unicity of the polarization form: if $(\mathscr{H}, \theta)$ is a $\mathrm{BT}_{1}$ with $\kappa$-action and $\mu_{1}, \mu_{2}: \mathscr{H} \rightarrow \mathscr{H}^{D}$ are two $\kappa$ - $\varepsilon$-dualities then $\left(\mathscr{H}, \mu_{1}, \theta\right) \cong\left(\mathscr{H}, \mu_{2}, \theta\right)$. 
Our proof will combine two results. First we shall analyze the group $U$ of automorphisms of a pair $(\mathscr{H}, \theta)$ which induce the identity on the canonical blocks. We show that, if $\operatorname{char}(k) \neq 2$, every $f \in U$ with $f^{\dagger}=f$ can be written as $f=g^{\dagger} g$; see 5.12 below. After that we show that, given a triple $(\mathscr{H}, \mu, \theta)$ as in (GPE'), we can choose ordered bases $\beta_{a}$ for the canonical blocks $B_{a}$ which are $F-V$-compatible and which are also "good" with respect to the pairings $\psi_{a}: B_{a} \times B_{\tilde{a}} \rightarrow k$; this is done in 5.13. Combining everything, the proof of the theorem is completed in 5.14 .

5.9. Lemma. - Let $k$ be an algebraically closed field of characteristic $\neq 2$. Let $G$ be a connected unipotent $k$-group (written multiplicatively), equipped with an anti-involution $g \mapsto g^{\dagger}$. Then every $f \in G$ with $f^{\dagger}=f$ can be written as $f=g^{\dagger} \cdot g$ for some $g \in G$.

Proof. There is an exact sequence $1 \longrightarrow G_{1} \longrightarrow G \stackrel{\pi}{\longrightarrow} V \longrightarrow 0$, with $V$ a non-zero vector group (written additively) and $G_{1} \subset G$ connected and stable under $\dagger$. Choose $x \in G$ with $\pi(x)=-\frac{1}{2} \pi(g)$. Then $g_{1}:=x^{\dagger} g x$ is an element of $G_{1}$ with $g_{1}^{\dagger}=g_{1}$. The lemma follows by induction on $\operatorname{dim}(G)$.

5.10. Let $(\mathscr{H}, \theta)$ be a $\mathrm{BT}_{1}$ with an action of $\kappa$. By our results in the previous section, the Dieudonné module $N$ is isomorphic to the standard object associated to $\underline{w}:=\underline{w}(\mathscr{H}, \theta)$. We shall work with the description of $N$ given in 5.7. As usual we view $N$ (via the given $\theta$ ) as a module over $K$. We identify $\operatorname{End}_{k}(\mathscr{H}, \theta)$ with the subring of $\operatorname{End}_{K}(N)=\prod_{i \in \mathscr{I}} \operatorname{End}_{\tilde{k}_{i}}\left(N_{i}\right)$ consisting of all endomorphisms which commute with $F$ and $V$.

Every $f \in \operatorname{End}_{k}(\mathscr{H}, \theta)$ stabilizes the canonical filtrations $C_{i} .$. For $a \in \mathscr{A}$ we can therefore define $\operatorname{gr}_{a}(f): B_{a} \rightarrow B_{a}$ as the homomorphism induced by $f$. Now consider the subgroup $U \subset \operatorname{Aut}_{k}(\mathscr{H}, \theta)$ given by

$$
U=\left\{f \in \operatorname{Aut}_{k}(\mathscr{H}, \theta) \mid \operatorname{gr}_{a}(f)=\operatorname{id}_{B_{a}} \text { for all } a \in \mathscr{A}\right\} .
$$

Let $\tilde{U}$ be the unipotent radical of the stabilizer of $\mathscr{C}$. inside the group $\operatorname{GL}_{K}(N)$; this $\tilde{U}$ is a connected unipotent $k$-group. By construction, $U$ is a subgroup of $\tilde{U}$.

Consider an element $f \in U$. Given $a, a^{\prime} \in \mathscr{A}$, let $P_{a, a^{\prime}}$ be the matrix of the composition

$$
B_{a} \hookrightarrow N \stackrel{f-\text { id }}{\longrightarrow} N \rightarrow B_{a^{\prime}}
$$

with respect to the bases $\beta_{a}$ and $\beta_{a^{\prime}}$. This is a matrix of size $d_{a^{\prime}} \times d_{a}$. Clearly we can have $P_{a, a^{\prime}} \neq 0$ only if $a^{\prime} \prec a$ (notation as in 4.14).

It is not so difficult to analyze the relations between the matrices $P_{a, a^{\prime}}$. To state the result, recall that each $t_{a}: B_{a} \rightarrow B_{\rho(a)}$ is called either an $F$-arrow or a $V$-arrow. Further let us write $\sigma:=$ Frob $_{k}: k \rightarrow k$. Now suppose that for each pair $\left(a, a^{\prime}\right) \in \mathscr{A} \times \mathscr{A}$ we have a matrix $P_{a, a^{\prime}}$ of size $d_{a^{\prime}} \times d_{a}$, with $P_{a, a^{\prime}} \neq 0$ only if $a^{\prime} \prec a$. Then a necessary and sufficient condition for this collection of matrices to come from an element $f \in U$ is that for all $\left(a, a^{\prime}\right)$ we have

$$
\begin{cases}P_{\rho(a), \rho\left(a^{\prime}\right)}=P_{a, a^{\prime}}^{\sigma} & \text { if } t_{a} \text { and } t_{a^{\prime}} \text { are of the same kind; } \\ P_{a, a^{\prime}}=0 & \text { if } t_{a} \text { is a } V \text {-arrow, } t_{a^{\prime}} \text { is an } F \text {-arrow. }\end{cases}
$$

(As we have already assumed that $P_{a, a^{\prime}}=0$ whenever $a^{\prime} \nprec a$, the only case where we get a new relation $P_{a, a^{\prime}}=0$ is when $a^{\prime} \prec a$ and $\rho\left(a^{\prime}\right) \prec \rho(a)$, with $t_{a}$ a $V$-arrow, $t_{a^{\prime}}$ an $F$-arrow. In the terminology of 4.14 this is the up-down case.)

At this point we use what we found in 4.14. Write $\mathscr{V}$ for the set of pairs $\left(a, a^{\prime}\right) \in \mathscr{A} \times \mathscr{A}$ such that $a^{\prime} \prec a$. Given such a pair $\left(a, a^{\prime}\right)$, consider the corresponding orbits $O$ and $O^{\prime}$. We 
mark these orbits such that $a(0)=a$ and $a^{\prime}(0)=a^{\prime}$. Let $\left[n_{1}, n_{2}\right]$ (with $n_{1} \leq 0 \leq n_{2}$ ) be the largest interval around 0 in which the marked orbits $O$ and $O^{\prime}$ are parallel. We say that $\left(b, b^{\prime}\right) \in \mathscr{V}$ is equivalent to $\left(a, a^{\prime}\right)$ if there exists an index $n \in\left[n_{1}, n_{2}\right]$ with $\left(b, b^{\prime}\right)=\left(a(n), a^{\prime}(n)\right)$. This defines an equivalence relation on $\mathscr{V}$. We say that the class of $\left(a, a^{\prime}\right)$ is a nill-class if the pair of orbits $\left(O, O^{\prime}\right)$ is up-down at index $n_{2}$. (See the illustration in 4.14.) Finally, we call the pair $\left(a\left(n_{1}\right), a^{\prime}\left(n_{1}\right)\right)$ the distinguished representative for the equivalence class of $\left(a, a^{\prime}\right)$.

With this terminology, (5.10.1) can be rephrased as follows. If the class of $\left(a, a^{\prime}\right)$ is a nillclass then we must have $P_{a, a^{\prime}}=0$. If the class is not a nill-class, with distinguished representative $\left(a\left(n_{1}\right), a^{\prime}\left(n_{1}\right)\right)$, then $P=P_{a\left(n_{1}\right), a^{\prime}\left(n_{1}\right)}$ can be chosen arbitrarily and

$$
P_{a, a^{\prime}}=P^{\sigma^{-n_{1}}} .
$$

In particular, this implies the following result.

5.11. Lemma. - The group $U$ is a connected unipotent subgroup of $\tilde{U}$.

5.12. Corollary - Assume char $(k) \neq 2$. Let $(\mathscr{H}, \mu, \theta)$ be a triple as in (GPE'). Write $f \mapsto f^{\dagger}$ for the Rosati involution on $\operatorname{End}_{k}(\mathscr{H}, \theta)$ induced by $\mu$, and notice that $U$ is stable under $\dagger$. Then every $f \in U$ with $f^{\dagger}=f$ can be written as $f=g^{\dagger} \cdot g$ for some $g \in U$.

Now we come to the final main ingredient of the proof of 5.5 .

5.13. Lemma. - Let $(\mathscr{H}, \mu, \theta)$ be a triple as in (GPE'). Choose constants $c(a)(a \in \mathscr{A})$ as in 5.7. Then for every $a \in \mathscr{A}$ we can choose an ordered basis $\beta_{a}$ for $B_{a}$ such that: (a) $\beta_{\rho(a)}$ is the image of $\beta_{a}$ under $t_{a}: B_{a} \rightarrow B_{\rho(a)}$ and (b) the form $\psi_{a}: B_{a} \times B_{\tilde{a}} \rightarrow k$ is given on the bases $\beta_{a}$ and $\beta_{\tilde{a}}$ by the matrix anti-diag $(c(a))$.

Proof. Given an orbit $O \subset \mathscr{A}$, write $\tilde{O}:=\{\tilde{a} \mid a \in O\}$, which is also an orbit. It suffices to find the bases $\beta_{a}$ for $a$ running through $O \cup \tilde{O}$. If $O \neq \tilde{O}$ this is easy: for $a \in O$ choose ordered bases $\beta_{a}$ satisfying condition (a). (See Lemma 4.10.) For $\tilde{a} \in \tilde{O}$ let $\beta_{\tilde{a}}$ then be the basis defined by condition (b). These again satisfy (a), and we are done.

Next suppose that $O=\tilde{O}$. Then $O$ has even length, say $2 s$. Fix a marking $n \mapsto a(n)$ of $O$. For $r \geq 1$ consider the Frob $_{k}^{r}$-linear isomorphisms

$$
T^{(r)}:=\left(B_{a(0)} \stackrel{t_{a(0)}}{\longrightarrow} B_{a(1)} \stackrel{t_{a(1)}}{\longrightarrow} \cdots \stackrel{t_{a(r-1)}}{\longrightarrow} B_{a(r)}\right)
$$

and

$$
U^{(r)}:=\left(B_{a(s)} \stackrel{t_{a(s)}}{\longrightarrow} B_{a(s+1)} \stackrel{t_{a(s+1)}}{\longrightarrow} \cdots \stackrel{t_{a(s+r-1)}}{\longrightarrow} B_{a(s+r)}\right) .
$$

Using the relation (5.7.1) we see that the diagram

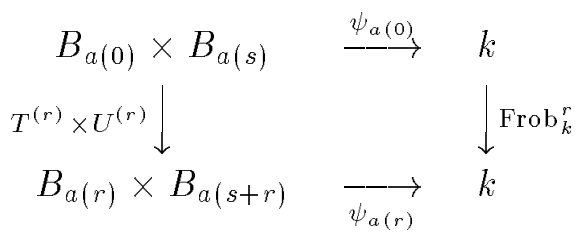

is commutative.

Consider the $\mathbb{F}_{p^{2 s} \text {-vector spaces }}$

$$
\mathscr{B}_{a(0)}:=\left\{b \in B_{a(0)} \mid T^{(2 s)}(b)=b\right\} \quad \text { and } \quad \mathscr{B}_{a(s)}:=\left\{b \in B_{a(s)} \mid U^{(2 s)}(b)=b\right\} .
$$


We have $\mathscr{B}_{a(0)} \otimes_{\mathbb{F}_{p} 2 s} k \stackrel{\sim}{\longrightarrow} B_{a(0)}$ and $\mathscr{B}_{a(s)} \otimes_{\mathbb{F}_{p} 2 s} k \stackrel{\sim}{\longrightarrow} B_{a(s)}$. The maps $T^{(s)}$ and $U^{(s)}$ restrict to Frob ${ }^{s}$-linear isomorphisms

$$
\mathscr{T}^{(s)}: \mathscr{B}_{a(0)} \stackrel{\sim}{\longrightarrow} \mathscr{B}_{a(s)} \quad \text { and } \quad \mathscr{U}^{(s)}: \mathscr{B}_{a(s)} \stackrel{\sim}{\longrightarrow} \mathscr{B}_{a(0)},
$$

inverse to each other. Taking $r=2 s$ in diagram (5.13.1) we find that $\psi_{a(0)}$ restricts to a perfect pairing $\mathscr{B}_{a(0)} \times \mathscr{B}_{a(s)} \longrightarrow \mathbb{F}_{p^{2 s}}$. Next taking $r=s$ in (5.13.1) we find that

$$
H: \mathscr{B}_{a(0)} \times \mathscr{B}_{a(0)} \longrightarrow \mathbb{F}_{p^{2 s}} \quad \text { defined by } \quad H\left(b, b^{\prime}\right)=\psi_{a(0)}\left(b, \mathscr{T}^{(s)}\left(b^{\prime}\right)\right)
$$

is an $\varepsilon$-symmetric hermitian form with respect to the involution Frob ${ }^{s}$ of $\mathbb{F}_{p^{2 s}}$ over $\mathbb{F}_{p^{s}}$. Notice that the discriminant of such a form is in $\mathbb{F}_{p^{s}}^{\times} / \operatorname{Norm}\left(\mathbb{F}_{p^{2 s}}^{\times}\right)$, which is the trivial group.

Essentially, all we have to do now is to bring $H$ in standard form. Note that the forms $H$ are classified by a cohomology group $H^{1}\left(\mathbb{F}_{p^{s}}, \mathrm{SU}\left(\mathscr{B}_{a(0)}, H\right)\right)$, and by Lang's theorem this group is trivial. (See Knus et al., [5], Section 29.19, and Serre [15], Chap. III, §2.) Hence we can choose a basis $\beta_{a(0)}$ on which the form $H$ is given by the matrix anti-diag $(c(a(0)))$. Now define $\beta_{a(n)}$ to be the image of $\beta_{a(0)}$ under $T^{(n)}$. This gives bases $\beta_{a}$ as required.

5.14. End of the proof. - Combining all previous results, let us now complete the proof of Theorem 5.5. We take a triple $(\mathscr{H}, \mu, \theta)$ as in (GPE'). Write $(N, F, V, \Psi, \theta)$ for the corresponding Dieudonné module with additional structure. Let $f \mapsto f^{\dagger}$ be the Rosati involution on $\operatorname{End}_{k}(\mathscr{H}, \theta)$.

Write $\underline{w}:=\underline{w}(\mathscr{H}, \mu, \theta)$. Choose constants $c(a)$, for $a \in \mathscr{A}$ as in 5.7. Write $N_{\underline{w}}=\oplus_{a \in \mathscr{A}} B_{a}$ for the standard Dieudonné module corresponding to $\underline{w}$; we use the description given in 5.7 , but in order to avoid confusion with the module $N$ we now do write the subscript “ $\underline{w}$ ". Let $\Psi_{\underline{w}}$ be the pairing on $N_{\underline{w}}$ constructed in loc. cit.

Choose bases $\beta_{a}$ as in Lemma 5.13. This gives us an isomorphism of Dieudonné modules with $\kappa$-action $h: N \stackrel{\sim}{\longrightarrow} N_{\underline{w}}$. Further, taking $h$ as an identification, the two pairings $\Psi$ and $\Psi_{\underline{w}}$ induce the same pairings $\psi_{a}: B_{a} \times B_{\tilde{a}} \rightarrow k$ on the canonical blocks. It follows that there is an element $f \in U$ with $f^{\dagger}=f$ such that $\Psi_{\underline{u}}\left(n_{1}, n_{2}\right)=\Psi\left(f n_{1}, n_{2}\right)$ for all $n_{1}, n_{2} \in N$. By Corollary 5.12 this $f$ can be written as $f=g^{\dagger} \cdot g$ for some $g \in U$. Then $h \circ g^{-1}: N \stackrel{\sim}{\longrightarrow} N_{\underline{w}}$ is an isomorphism which is compatible both with the $k$-actions and the pairings. The existence of such an isomorphism is what we had to prove.

\section{6. $\mathrm{BT}_{1}$ 's with endomorphisms and a polarization - case A}

In this section we deal with problem (GPE) stated in 5.1 in case the algebra with involution $(D, *)$ is simple of type (A). There are some new features, but once we have set up notations most steps in the proof of Theorem 6.7 are the same as in the previous section. Therefore we shall explain the new features and state the main result, but only outline the proof.

6.1. The setup. - We consider a semi-simple algebra $D$ with involution $*$ of the second kind; as explained in 5.1 we may assume that $(D, *)$ is simple as an algebra with involution. Write $\tilde{\kappa}$ for the center of $D$ and $\kappa:=\left\{a \in \tilde{\kappa} \mid a^{*}=a\right\}$. Then $\kappa$ is a finite field and either $\tilde{\kappa}=\kappa \times \kappa$, which we call case (A1), or $\tilde{\kappa}$ is a quadratic field extension of $\kappa$, called case (A2).

Set $\mathscr{I}:=\operatorname{Hom}(\kappa, k)$ and $\tilde{\mathscr{I}}:=\operatorname{Hom}(\tilde{\kappa}, k)$. We shall typically use the letter $i$ for an element of $\mathscr{I}$ and $\tau$ for an element of $\widetilde{\mathscr{I}}$. For $i \in \mathscr{I}$, set $i+1:=$ Frob $_{k} \circ i$. For $\tau \in \widetilde{\mathscr{I}}$, set $\tau+1=$ Frob $_{k} \circ \tau$ and $\bar{\tau}=\tau \circ *$. In case (A2), taking $\tau+1$ as the successor of $\tau$ gives $\widetilde{\mathscr{I}}$ a cyclic ordering. We have 
$\bar{\tau}=\tilde{\tau}$, using the notation of 4.1. Similarly, if in case (A1) we impose the condition that $\bar{\tau}=\tilde{\tau}$ then we have a natural bi-cyclic ordering on $\tilde{\mathscr{I}}$. In both cases the set $\mathscr{I}$ has a cyclic ordering. We have a natural $2: 1$ map $\widetilde{\mathscr{I}} \rightarrow \mathscr{I}$; if this sends $\tau \in \widetilde{\mathscr{I}}$ to $i \in \mathscr{I}$ then we refer to $\tau$ and $\bar{\tau}$ as the elements of $\tilde{\mathscr{I}}$ lying over $i$.

For $i \in \mathscr{I}$ and $\tau \in \widetilde{\mathscr{I}}$ we write

$$
k_{\tau}:=\tilde{\kappa} \underset{\tilde{\kappa}, \tau}{\otimes} k, \quad k_{i}:=\kappa \underset{\kappa, i}{\otimes} k, \quad \text { and } \quad \tilde{k}_{i}:=\tilde{\kappa}_{\kappa, i} \otimes .
$$

Let

$$
K:=\kappa \otimes_{\mathbb{T}_{p}} k=\prod_{i \in \mathscr{I}} k_{i} \quad \text { and } \quad \tilde{K}:=\tilde{\kappa} \otimes_{\mathbb{F}_{p}} k=\prod_{i \in \mathscr{I}} \tilde{k}_{i}=\prod_{\tau \in \tilde{I}} k_{\tau} .
$$

The maps id $\otimes$ Frob $_{k}$ give Frob $k$-linear isomorphisms

$$
F_{k_{\tau}}: k_{\tau} \rightarrow k_{\tau+1}, \quad F_{k_{i}}: k_{i} \rightarrow k_{i+1}, \quad F_{\tilde{k}_{i}}: \tilde{k}_{i} \rightarrow \tilde{k}_{i+1}, \quad F_{K}: K \rightarrow K, \quad F_{\tilde{K}}: \tilde{K} \rightarrow \tilde{K} .
$$

6.2. Morita equivalence. - Similar to what was done in 5.2, we can reduce problem (GPE) to a problem with $D=\tilde{\kappa}$. This time, the category $\mathbf{C}_{1}$ is equivalent to the category $\mathbf{C}_{2}$ of 5-tuples $\left(N, F_{N}, V_{N}, \Psi, \theta\right)$, where

- $\left(N, F_{N}, V_{N}\right)$ is the Dieudonné module of a $\mathrm{BT}_{1}$;

- $\theta: \tilde{\kappa} \rightarrow \operatorname{End}_{\mathrm{DM}}(N)$ defines an action of $\tilde{\kappa}$ on $N$, making it a free module over $\tilde{K}$;

- $\Psi: N \times N \rightarrow \tilde{K}$ is an alternating hermitian pairing with respect to the non-trivial automorphism of $\tilde{K}$ over $K$.

Note that we only consider modules $N$ which are free over $\tilde{K}$. To explain why, recall from 4.3 that our modules $N$ are free over $K$. As we shall see below, the existence of a hermitian pairing with values in $\tilde{K}$ implies that they are even free over $\tilde{K}$.

Translating back to group schemes the problem becomes the following.

(GPE') Classification of triples $(\mathscr{H}, \mu, \theta)$, where $(\mathscr{H}, \theta)$ is a $\mathrm{BT}_{1}$ with an action of $\tilde{\kappa}$, and where $\mu: \mathscr{H} \stackrel{\sim}{\longrightarrow} \mathscr{H}^{D}$ is a $\tilde{\kappa}$-(-1)-duality.

Here we use the ad hoc term " $\tilde{\kappa}-(-1)$-duality" for a duality $\mu$ corresponding to an alternating hermitian form $\Psi$ on the Dieudonné module.

6.3. Let $\left(N, F_{N}, V_{N}, \Psi, \theta\right)$ be an object of the category $\mathbf{C}_{2}$. As in 4.3 , the action of $\kappa$ gives rise to a decomposition $N=\oplus_{i \in \mathscr{I}} N_{i}$. Using the full action of $\tilde{\kappa}$ this is refined to s decomposition $N=\oplus_{\tau \in \widetilde{I}} N_{\tau}$. If $\tau$ and $\bar{\tau}$ are the two elements of $\widetilde{\mathscr{I}}$ lying over $i \in \mathscr{I}$ then $N_{i}=N_{\tau} \oplus N_{\bar{\tau}}$.

Frobenius and Verschiebung become homomorphisms $F_{N_{\tau}}: N_{\tau} \rightarrow N_{\tau+1}$ and $V_{N_{\tau}}: N_{\tau} \leftarrow$ $N_{\tau+1}$, equivariant with respect to $F_{k_{\tau}}: k_{\tau} \rightarrow k_{\tau+1}$, respectively its inverse.

By a similar procedure as in 4.4 , we obtain canonical filtrations

$$
C_{\tau,:}: \quad(0)=C_{\tau, 0} \subsetneq C_{\tau, 1} \subsetneq \cdots \subsetneq C_{\tau, \ell_{\tau}}=N_{\tau}
$$

By construction, for each $\tau \in \widetilde{I}$ there is an index $h_{\tau} \in\left\{0,1, \ldots, \ell_{\tau}\right\}$ such that $C_{\tau, h_{\tau}}=$ $\operatorname{Im}\left(F_{N_{\tau-1}}\right)$. The analogue of Lemma 4.5 in this setting is as follows.

6.4. Lemma. - (0) The integer $\ell:=\ell_{\tau}$ is independent of $\tau \in \tilde{\mathscr{I}}$.

(i) For each $\tau \in \widetilde{\mathscr{I}}$ and $j \in\{0,1, \ldots, \ell\}$ there exist indices $r(j)=r_{\tau}(j) \in\left\{0,1, \ldots, h_{\tau+1}\right\}$ and $s(j)=s_{\tau}(j) \in\left\{h_{\tau+1}, \ldots, \ell\right\}$ such that

$$
F_{N_{\tau}}\left(C_{\tau, j}\right)=C_{\tau+1, r(j)} \quad \text { and } \quad V_{N_{\tau}}^{-1}\left(C_{\tau, j}\right)=C_{\tau+1, s(j)}
$$


(ii) For $j \geq 1$, either

$$
C_{\tau, j} \cap \operatorname{Ker}\left(F_{N_{\tau}}\right)=C_{\tau, j-1} \cap \operatorname{Ker}\left(F_{N_{\tau}}\right)
$$

or

$$
C_{\tau, j} \subset \operatorname{Span}\left(C_{\tau, j-1}, \operatorname{Ker}\left(F_{N_{\tau}}\right)\right)
$$

In the first case we have $r_{\tau}(j)=r_{\tau}(j-1)+1$ and $s_{\tau}(j)=s_{\tau}(j-1)$; in the second case $r_{\tau}(j)=r_{\tau}(j-1)$ and $s_{\tau}(j)=s_{\tau}(j-1)+1$. Further,

$$
C_{\tau, j} \cap \operatorname{Ker}\left(F_{N_{\tau}}\right)=C_{\tau, j-1} \cap \operatorname{Ker}\left(F_{N_{\tau}}\right) \quad \Longleftrightarrow \quad C_{\bar{\tau}, \ell+1-j} \subset \operatorname{Span}\left(C_{\bar{\tau}, \ell-j}, \operatorname{Ker}\left(F_{N_{\bar{\tau}}}\right)\right) .
$$

(iii) For all $\tau$ and $j$ we have $r_{\tau}(j)+s_{\tau}(j)=\ell+j$ and $r_{\tau}(j)+s_{\bar{\tau}}(\ell-j)=\ell$.

6.5. We define the canonical blocks by

$$
B_{\tau, j}:=C_{\tau, j} / C_{\tau, j-1} \quad\left(\text { for } \tau \in \widetilde{\mathscr{I}} \text { and } j \in\left\{1, \ldots, \ell_{\tau}\right\}\right)
$$

Further we have permutations $\rho_{\tau} \in \mathfrak{S}_{\ell}$, given by

$$
\rho_{\tau}(j)= \begin{cases}r_{\tau}(j) & \text { if } C_{\tau, j} \cap \operatorname{Ker}\left(F_{N_{\tau}}\right)=C_{\tau, j-1} \cap \operatorname{Ker}\left(F_{N_{\tau}}\right) \\ s_{\tau}(j) & \text { if } C_{\tau, j} \subset \operatorname{Span}\left(C_{\tau, j-1}, \operatorname{Ker}\left(F_{N_{\tau}}\right)\right) .\end{cases}
$$

As in 4.9 we obtain, for $\tau \in \widetilde{\mathscr{I}}$ and $j \in\{1,2, \ldots, \ell\}$, a Frob ${ }_{k}$-linear isomorphism

$$
t_{\tau, j}: B_{\tau, j} \stackrel{\sim}{\longrightarrow} B_{\tau+1, \rho_{\tau}(j)},
$$

induced either by Frobenius or (inversely) by Verschiebung.

We have an involution $(a, b) \mapsto(b, a)$ of $\tilde{k}_{i} \cong k_{\tau} \times k_{\bar{\tau}}=k \times k$ over $k_{i}=k$. We shall write this automorphism as $x \mapsto \bar{x}$. Note that if we restrict this automorphism to one of the two factors we get an isomorphism $k_{\tau} \rightarrow k_{\bar{\tau}}$ which, via the identifications $k_{\tau}=k=k_{\bar{\tau}}$, is just the identity on the field $k$; in other words: if $a \in k_{\tau}$ then $\bar{a}$ is the same element of $k$, but now viewed of an element of $k_{\bar{\tau}}$. The map $k_{\tau} \rightarrow k_{\bar{\tau}}$ given by $a \mapsto \bar{a}$ should be thought of as "complex conjugation"; in particular, if $C_{\tau}$ is a $k_{\tau}$-module and $C_{\bar{\tau}}$ is a $k_{\bar{\tau}}$-module then it makes sense to say that a pairing $\psi: C_{\tau} \times C_{\bar{\tau}} \rightarrow k_{\tau}$ is anti-linear in the second variable.

With this terminology, we have perfect pairings, linear in the first variable, anti-linear in the second variable,

$$
\psi_{\tau}: N_{\tau} \times N_{\bar{\tau}} \rightarrow k_{\tau} \quad \text { with } \quad \psi_{\bar{\tau}}(y, x)=-\overline{\psi_{\tau}(x, y)} .
$$

In particular, $\operatorname{dim}\left(N_{\tau}\right)=\operatorname{dim}\left(N_{\bar{\tau}}\right)$, and it follows that the $\tilde{K}$-module $N$ is free, as claimed in 6.2 .

The canonical filtrations $C_{\tau}$, and $C_{\bar{\tau}, \bullet}$ are dual with respect to the pairings $\psi_{\tau}$ and $\psi_{\bar{\tau}}$. In particular, on the canonical blocks we get induced perfect pairings

$$
\bar{\psi}_{\tau, j}: B_{\tau, j} \times B_{\bar{\tau}, \ell+1-j} \longrightarrow k_{\tau}
$$

6.6. Definition of the element $\underline{w}(\mathscr{H}, \mu, \theta)$. - Let $(\mathscr{H}, \mu, \theta)$ be a triple as in (GPE'). Write $\left(N, F_{N}, V_{N}, \Psi, \theta\right)$ for the corresponding Dieudonné module. Let $q$ be the $\tilde{K}$-rank of $N$. For $\tau \in \widetilde{\mathscr{I}}$, let $\mathfrak{f}(\tau)$ be the $k$-dimension of $N[F]_{\tau}:=\operatorname{Ker}\left(F_{N_{\tau}}\right)$. This defines a function $\mathfrak{f}: \widetilde{\mathscr{I}} \rightarrow$ $\{0,1, \ldots, q\}$ with the property that $\mathfrak{f}(\tau)+\mathfrak{f}(\bar{\tau})=q$ for all $\tau$. 
Let $\Phi: \tilde{K}^{q} \times \tilde{K}^{q} \rightarrow \tilde{K}$ be an alternating hermitian form, $\tilde{K}$-linear in the first variable, $\tilde{K}$ antilinear in the second. Write $G=\mathrm{U}_{\tilde{K}}\left(\tilde{K}^{q}, \Phi\right)$ for the group of $\tilde{K}$-linear unitary automorphisms. The pairing $\Phi$ is the product of pairings

$$
\varphi_{i}=\left(\varphi_{\tau}, \varphi_{\bar{\tau}}\right): \tilde{k}_{i}^{q} \times \tilde{k}_{i}^{q}=\left(k_{\tau}^{q} \oplus k_{\bar{\tau}}^{q}\right) \times\left(k_{\tau}^{q} \oplus k_{\bar{\tau}}^{q}\right) \longrightarrow \tilde{k}_{i}=k_{\tau} \times k_{\bar{\tau}}
$$

(where $\varphi_{\tau}$ pairs $k_{\tau}^{q}$ with $k_{\bar{\tau}}^{q}$ ), and we have $G=\prod_{i \in \mathscr{I}} G_{i}$, with $G_{i}=\operatorname{Aut}_{\tilde{k}_{i}}\left(k_{i}^{2 q}, \varphi_{i}\right)$. If $\tau$ and $\bar{\tau}$ are the two elements of $\widetilde{\mathscr{I}}$ lying over $i \in \mathscr{I}$ then we have isomorphisms

$$
\mathrm{GL}_{q, k_{\tau}} \stackrel{\sim}{\longrightarrow} G_{i} \stackrel{\sim}{\longrightarrow} \mathrm{GL}_{q, k_{\bar{\tau}}}
$$

Choose an isometry $\xi:(N, \Psi) \stackrel{\sim}{\longrightarrow}\left(\tilde{K}^{q}, \Phi\right)$ of hermitian $\tilde{K}$-modules; this allows us to view the $N[F]_{\tau}$ and $C_{\tau}$, as partial flags in $k_{\tau}^{q}$. Viewing $k_{\bar{\tau}}^{q}$ as the dual of $k_{\tau}^{q}$ via the perfect pairing $\psi_{\tau}$ we have $N[F]_{\bar{\tau}}=N[F]_{\tau}^{\perp}$ and $C_{\bar{\tau}, \bullet}=C_{\tau}^{\perp} \bullet$

Write $P:=\operatorname{Stab}_{G}(N[F])$ and $Q:=\operatorname{Stab}_{G}\left(\mathscr{C}_{\bullet}\right)$. Let $W=W_{G}$, and write $X$ for the type of $P$. We have a natural decomposition $W_{X} \backslash W=\prod_{i \in \mathscr{I}} W_{X_{i}} \backslash W_{i}$. The inclusion map $G \hookrightarrow \mathrm{GL}_{q, \tilde{K}}$ gives rise to an injective map

$$
W_{X} \backslash W \longleftrightarrow W_{X_{\mathrm{f}}} \backslash W_{\mathrm{GL}_{q, \tilde{K}}},
$$

writing $X_{\mathrm{f}}$ for the type of the stabilizer of $N[F]$ in $\mathrm{GL}_{q, \tilde{K}}$ (which is equivalent to knowing the function $\mathfrak{f}$ ).

Working as in 4.6 and 5.4, using (ii) of Lemma 6.4 and what was explained in Examples 3.5 and 3.11 , we obtain a well-defined element

$$
\underline{w}(\mathscr{H}, \mu, \theta):=w^{\sharp}(P, Q) \in W_{X} \backslash W,
$$

independent of the choice of $\xi$.

6.7. Theorem. - Notations as above. Assume that char $(k) \neq 2$.

(i) Let $(\mathscr{H}, \theta)$ be a $B T_{1}$ with an action of $\tilde{\kappa}$, of type $(q, \mathfrak{f})$. Let $\underline{w}(\mathscr{H}, \theta)$ be the associated element of $\mathscr{W}\left(\mathrm{GL}_{q, \tilde{K}} ; X_{\mathrm{f}}\right)$. Then there exists a $\tilde{\kappa}-(-1)$-duality $\mu: \mathscr{H} \rightarrow \mathscr{H}^{\vee}$ if and only if $\underline{w}(\mathscr{H}, \theta)$ is in the image of the map (6.6.1).

(ii) If a duality $\mu$ as in (i) exists then it is unique up to isomorphism. In other words: sending a triple $(\mathscr{H}, \mu, \theta)$ as in (GPE') to the element $\underline{w}(\mathscr{H}, \mu, \theta) \in \mathscr{W}(G ; X)$ gives a bijection

$$
\left\{\begin{array}{c}
\text { isomorphism classes of triples } \\
(\mathscr{H}, \mu, \theta) \text { of type }(q, \mathfrak{f})
\end{array}\right\} \stackrel{\sim}{\longrightarrow} \mathscr{W}(G ; X) .
$$

As explained in 3.11, if $W_{\tau}$ is the Weyl group of $\mathrm{GL}_{q, k_{\tau}}$ and $X_{\tau}$ is the type of the stabilizer of $N[F]_{\tau}$ then we have natural bijections

$$
W_{X_{\tau}} \backslash W_{\tau} \stackrel{\sim}{\longrightarrow} W_{X_{\bar{\tau}}} \backslash W_{\bar{\tau}} \quad \text { denoted } \pi \mapsto \check{\pi}
$$

Then the set $\mathscr{W}(G ; X)$ can be described as

$$
\mathscr{W}(G ; X)=\left\{\left(\pi_{\tau}\right)_{\tau \in \tilde{\mathscr{I}}} \mid \pi_{\tau} \in W_{X_{\tau}} \backslash W_{\tau} \text { and } \pi_{\bar{\tau}}=\check{\pi}_{\tau} \text { for all } \tau .\right\}
$$

6.8. Remark. - Suppose we are in case (A1), meaning that $\tilde{\kappa}=\kappa \times \kappa$ with involution $\left(x_{1}, x_{2}\right) \mapsto\left(x_{2}, x_{1}\right)$. Let us number the two factors $\kappa$ as $\kappa^{(1)}$ and $\kappa^{(2)}$. The set $\tilde{\mathscr{I}}$ naturally 
decomposes, as a set with bi-cyclic ordering, as $\widetilde{I}=\mathscr{I}^{(1)} \mathrm{II} \mathscr{I}^{(2)}$. Write $\mathfrak{f}^{(\nu)}$ : $\mathscr{I}=\mathscr{I}^{(\nu)} \longrightarrow$ $\{1, \ldots, q\}$ for the restriction of $\mathfrak{f}$ to $\mathscr{I}^{(\nu)}$.

For $\nu=1,2$, let $\left(\mathscr{H}^{(\nu)}, \theta^{(\nu)}\right)$ be a $\mathrm{BT}_{1}$ with $\kappa$-action, of type $\left(q, f^{(\nu)}\right)$, with $\left(\mathscr{H}^{(\nu)}, \theta^{(\nu)}\right)$ dual to $\left(\mathscr{H}^{(\nu+1)}, \theta^{(\nu+1)}\right)$. Then take $(\mathscr{H}, \theta):=\left(\mathscr{H}^{(1)}, \theta^{(1)}\right) \times\left(\mathscr{H}^{(2)}, \theta^{(2)}\right)$, which comes with a natural action of $\tilde{\kappa}$. Take

$$
\mu=\left(\begin{array}{cc}
0 & \zeta \\
-\zeta^{D} & 0
\end{array}\right), \text { where } \zeta:\left(\mathscr{H}^{(1)}, \theta^{(1)}\right) \stackrel{\sim}{\longrightarrow}\left(\mathscr{H}^{(2)}, \theta^{(2)}\right)
$$

This gives us a triple $(\mathscr{H}, \mu, \theta)$ as in (GPE'), and every such triple is of this form. One verifies that Theorems 4.7 and 6.7 are consistent.

6.9. Outline of the proof. - The proof of 6.7 is essentially the same as that of Theorem 5.5. By the previous remark we may assume that $\tilde{\kappa}$ is a field.

The first step is to show that if $\underline{w}$ is an element in the image of the map (6.6.1), there exists a $\tilde{\kappa}-(-1)$-duality on the standard object $\left(\mathscr{H}_{\underline{w}}, \theta_{\underline{w}}\right)$. For the set $\mathscr{A}$ we now take $\mathscr{A}:=\widetilde{\mathscr{I}} \times\{1, \ldots, \ell\}$, with permutation $\rho$ given by $\rho(\tau, j)=\left(\tau+1, \rho_{\tau}(j)\right)$. For $a=(\tau, j) \in \mathscr{A}$ we set $\tilde{a}:=(\bar{\tau}, \ell+1-j)$. Then we proceed exactly as in 5.7 , noting that the desired pairings are now hermitian pairings $\psi_{a}: B_{a} \times B_{\tilde{a}} \rightarrow k$.

After that we need to prove that the $\tilde{\kappa}$ - $(-1)$-duality is unique (up to isomorphism). The arguments in 5.10 do not use any polarization, so that Corollary 5.12 is still valid. Thus only Lemma 5.13 remains. Our notations have been set up in such a way that we can copy the proof in 5.13 verbatim.

6.10. An example: the CM-case. - To illustrate the use of the "multiplication type" $f$, and to give a brief preview of what will be done in [10], let us show how to compute the $p$-kernel of a reduction of an abelian variety of CM-type. This is very similar to the recipe for computing the Newton polygon of such a reduction, which we shall recall.

Let $F$ be a CM-field of degree $2 g$ over $\mathbb{Q}$, i.e., a totally imaginary quadratic extension of a totally real field $F_{0}$. Given an embedding $\sigma: F \rightarrow \mathbb{C}$, write $\bar{\sigma}$ for the conjugate embedding. By a $C M$-type for $F$ we mean a subset $\mathfrak{f} \subset \operatorname{Hom}(F, \mathbb{C})$ such that precisely one of each pair $(\sigma, \bar{\sigma})$ is in $\mathfrak{f}$.

Let $Y$ be an abelian variety of dimension $g$ over a number field $E \subset \mathbb{C}$. Suppose $Y$ is equipped with an action by an order $\mathscr{O} \subset F$; this gives us a homomorphisms $F \rightarrow \operatorname{End}(Y) \otimes \mathbb{Q}$. The first homology $H=H_{1}\left(Y_{\mathbb{C}}, \mathbb{C}\right)$ is free of rank 1 as a module over

$$
F \otimes \mathbb{Q}=\prod_{\sigma \in \operatorname{Hom}(F, \mathbb{C})} \mathbb{C}^{(\sigma)}
$$

Hodge theory gives us a canonical $\left(F \otimes_{\mathbb{Q}} \mathbb{C}\right)$-submodule $F^{0} H \subset H$, and we define $\mathfrak{f} \subset \operatorname{Hom}(F, \mathbb{C})$ to be the set of weights of this submodule. Then $f$ is a CM-type and we say that $Y$ is of CM-type $(F, f)$.

Choose embeddings $\overline{\mathbb{Q}} \hookrightarrow \overline{\mathbb{Q}}_{p} \hookrightarrow \mathbb{C}$. Let $\mathfrak{p}$ be the corresponding place of $E$ above $p$. Possibly after replacing $E$ by a finite extension, $Y$ has good reduction $Y_{0}$ over $k(\mathfrak{p})$. Via the chosen field embeddings we can identify

$$
\operatorname{Hom}(F, \mathbb{C})=\operatorname{Hom}\left(F, \overline{\mathbb{Q}}_{p}\right)=\operatorname{Hom}\left(F \otimes \mathbb{Q} \mathbb{Q}_{p}, \overline{\mathbb{Q}}_{p}\right)=\amalg_{\pi} \operatorname{Hom}\left(F_{\pi}, \overline{\mathbb{Q}}_{p}\right),
$$

where $\pi$ runs through the set of places of $F$ above $p$ and we write $F_{\pi}$ for the $\pi$-adic completion of $F$. The set $\mathfrak{f}$ is then a disjoint union of sets $\mathfrak{f}_{\pi} \subset \operatorname{Hom}\left(F_{\pi}, \overline{\mathbb{Q}}_{p}\right)$. 
In Tate's paper [16] it is explained how to compute the Newton polygon of $Y_{0}$. Namely, the slopes that occur are just the numbers $\# \mathfrak{f}_{\pi} /\left[F_{\pi}: \mathbb{Q}_{p}\right]$.

Write $k$ for the residue field of the maximal unramified subfield $\mathbb{Q}_{p}^{\text {nr }} \subset \overline{\mathbb{Q}}_{p}$; this $k$ is an algebraically closed field containing $k(\mathfrak{p})$. Assume that the order $\mathscr{O}$ is maximal at $p$ and that $p$ does not ramify in $F$. (This is what the usual conditions for being a prime of good reduction mean in the present case.) Then in a similar spirit we can also compute the isomorphism type of the $p$-kernel $Y_{0}[p]$ over $k$. Note that $\mathscr{O} / p \mathscr{O}$ is the product of the residue fields $k(\pi)$, and that the Dieudonné module $N$ of $Y_{0}[p]$ is free of rank 1 over $\mathscr{O} / p \mathscr{O}$. In particular, the structure of $Y_{0}[p]_{k}$ with its action of $\mathscr{O} / p \mathscr{O}$ is completely determined by the multiplication type $\mathfrak{f}_{0}: \mathscr{O} / p \mathscr{O} \rightarrow k$. Now the assumption that $p$ is unramified in $F$ gives us a natural identification

$$
\operatorname{Hom}\left(F \otimes \mathbb{Q}_{p}, \overline{\mathbb{Q}}_{p}\right)=\operatorname{Hom}\left(F \otimes \mathbb{Q}_{p}, \mathbb{Q}_{p}^{\text {nr }}\right) \stackrel{\sim}{\longrightarrow} \operatorname{Hom}(\mathscr{O} / p \mathscr{O}, k)
$$

and by looking at the Dieudonné module of the full $p$-divisible group $Y\left[p^{\infty}\right]$ we readily find that $\mathfrak{f}_{0}$ is just the image of $\mathfrak{f}$ under this map.

In concrete terms, compute the multiplication type $\mathfrak{f}_{0} \subset \mathscr{I}:=\operatorname{Hom}(\mathscr{O} / p \mathscr{O}, k)$ as the image of the type $\mathfrak{f}$. Given $i \in \mathscr{I}$, draw an arrow $(i) \stackrel{V}{\longleftarrow}(i+1)$ if $i \in \mathfrak{f}_{0}$, and arrow $(i) \stackrel{F}{\longrightarrow}(i+1)$ if not. This gives us a union of circular diagrams $\Gamma_{\pi}$ as described in 2.1 (one diagram, of size $\left[F_{\pi}: \mathbb{Q}_{p}\right]$, for each prime $\pi$ ), with a natural action of $\mathscr{O} / p \mathscr{O}$ (letting $x$ act as multiplication by $i(x)$ on the base vector corresponding to $i$.) This describes the $p$-kernel of $Y_{0}$.

\section{References}

[1] K.S. Brown, Buildings, Springer-Verlag, New York, 1989.

[2] N. Bourbaki, Groupes et Algèbres de Lie, Chap. 4-6, Masson, Paris, 1981.

[3] G. van der Geer, Cycles on the Moduli Space of Abelian Varieties, in: Moduli of Curves and Abelian Varieties, C. Faber and E. Looijenga, eds., Aspects of Mathematics E 33, Vieweg, Braunschweig/Wiesbaden, 1999, pp. 65-89.

[4] M.-A. Knus, Quadratic and Hermitian Forms over Rings, Grundlehren der mathematischen Wissenschaften 294, Springer-Verlag, Berlin, 1991.

[5] M.-A. Knus et al., The Book of Involutions, Colloquium Publ. 44, A.M.S., Providence, R.I., 1998.

[6] M. Demazure, Lectures on p-divisible groups, Lecture Notes in Math. 302, Springer-Verlag, Berlin, 1972.

[7] E.Z. Goren and F. Oort, Stratifications of Hilbert Modular Varieties, J. Alg. Geom. 9 (2000), 111-154.

[8] R.E. Kottwitz, Points on some Shimura Varieties over Finite Fields, Journal of the A.M.S. 5 (1992), 373-444.

[9] H. Kraft, Kommutative algebraische p-Gruppen (mit Anwendungen auf p-divisible Gruppen und abelsche Varietäten, manuscript, University of Bonn, September 1975, 86 pp.

[10] B.J.J. Moonen, The Ekedahl-Oort Stratification for PEL-type Moduli Spaces, in preparation.

[11] A. Ogus, Hodge Cycles and Crystalline Cohomology, in: Hodge Cycles, Motives, and Shimura Varieties, P. Deligne et al., Lecture Notes in Math. 900, Springer-Verlag, Berlin, 2nd printing, 1989 . 
[12] F. Oort, A Stratification of a Moduli Space of Polarized Abelian Varieties in Positive Characteristic, in: Moduli of Curves and Abelian Varieties, C. Faber and E. Looijenga, eds., Aspects of Mathematics E 33, Vieweg, Braunschweig/Wiesbaden, 1999, pp. 47-64.

[13] F. Oort, A Stratification of a Moduli Space of Abelian Varieties, manuscript, January 2000; to appear.

[14] M. Raynaud, Schémas en groupes de type $(p, \ldots, p)$, Bull. Soc. math. France 102 (1974), $241-280$.

[15] J.-P. Serre, Cohomologie Galoisienne, Lecture Notes in Math. 5, Springer-Verlag, Berlin, 5th ed., 1994.

[16] J. Tate, Classes d'Isogenie des Variétés Abéliennes sur un Corps Fini, Sém. Bourbaki, exp. 352, Novembre 1968, in: Lecture Notes in Math. 179, Springer-Verlag, Berlin, 1971.

[17] J. Tits Buildings of Spherical Type and Finite BN-Pairs, Lecture Notes in Math. 386, Springer-Verlag, Berlin, 2nd printing, 1986.

Ben Moonen, University of Utrecht, Department of Mathematics, P.O. Box 80.010, NL-3508 TA, Utrecht, The Netherlands. Email: moonen@math.uu.nl 